\title{
Localization of Atiyah classes
}

\author{
Marco Abate, Filippo Bracci, Tatsuo Suwa and Francesca Tovena
}

\begin{abstract}
We construct the Atiyah classes of holomorphic vector bundles using $(1,0)$-connections and developing a Chern-Weil type theory, allowing us to effectively compare Chern and Atiyah forms. Combining this point of view with the Cech-Dolbeault cohomology, we prove several results about vanishing and localization of Atiyah classes, and give some applications. In particular, we prove a Bott type vanishing theorem for (not necessarily involutive) holomorphic distributions. As an example we also present an explicit computation of the residue of a singular distribution on the normal bundle of an invariant submanifold that arises from the Camacho-Sad type localization.
\end{abstract}

\section{Introduction}

Characteristic classes are invariants of manifolds providing obstructions to the existence of certain geometric objects. For instance, it is well known that the existence of a nonsingular vector field on a manifold $M$ implies the vanishing of the top Chern class of $M$. Roughly speaking, characteristic classes detect the necessary existence of singularities (e.g., zeros of a vector field) of the given geometric object; and thus it is only natural to expect that, in a suitable sense, characteristic classes might be localized near those singularities. Residue (or index) theory systematically deals with such a localization procedure, and the results yield interesting and deep information both on the manifold and on the geometric object.

This approach has been developed with considerable success for Chern classes and singular holomorphic foliations, starting with the seminal work of P. Baum and R. Bott [6], up to very recent applications. It is based on the Bott vanishing theorem, which says that the characteristic forms associated to certain Chern polynomials vanish in the presence of actions of (or of partial connections along) nonsingular holomorphic foliations, i.e., involutive subbundles of the holomorphic tangent bundle. This suggests that in general the corresponding characteristic

Mathematics Subject Classification (2010): 14C17, 32A27, 32S65, 37F75, 53C05, 57R20, 58A30. Keywords: Atiyah forms and classes, connections of type (1,0), Čech-Dolbeault cohomology, localization and residues, Bott type vanishing, singular holomorphic distributions. 
classes should localize at the singular sets of holomorphic foliations, and thus it should be possible to express them using (suitably defined) residues.

In this way important index theorems have been proved; for the normal sheaf of a foliation (using Baum-Bott residues; see, e.g., [5], [6], and [13]), for the normal sheaf of invariant subvarieties (using Camacho-Sad residues; see, e.g., [12], [17], and [18]), and for the normal sheaf of the foliation along an invariant subvariety (using the so-called variation residues; see, e.g., [23]). In turn, these index theorems had important applications to dynamics. Just to give a few of examples, the Camacho-Sad index theorem is crucial in the proof by C. Camacho and P. Sad of the existence of separatrices for germs of singular holomorphic vector fields in $\mathbb{C}^{2}$ (see [12]); or, very recently, it has been effectively used by D. Marín and J.-F. Mattei in their topological classification of generic holomorphic foliations in $\mathbb{C}^{2}$ nearby a singular point (see [19]). We also cite [10] as a work giving diverse applications of these residue theorems.

Localization of Chern classes has proved to be useful in discrete dynamics too. For instance, in [1] (see also [8]) an analogue for holomorphic self-maps of the Camacho-Sad theorem is proved and applied to show the existence of parabolic curves for germs of holomorphic self-maps tangent to the identity in $\mathbb{C}^{2}$; and analogues for holomorphic self-maps, valid in any dimension, of Baum-Bott, Camacho-Sad, and variation residue theorems, with further applications to discrete dynamics, have been given in [2]. Furthermore, in [3] it is shown how these results, both for foliations and for self-maps, fit into a unified theory which can be described in the framework of the residue theory of partial holomorphic connections.

It should also be mentioned that localization techniques can also be applied to the study of characteristic classes of singular varieties, as summarized in [9]. The residue theory in this framework leads to an analytic intersection theory on singular varieties [24]. See also [22] for another development in this direction.

The present paper studies the localization of Atiyah classes of holomorphic vector bundles. Such classes have been introduced by M. Atiyah in [4] as Dolbeault cohomology classes providing obstructions to the existence of holomorphic connections on a given holomorphic vector bundle. Since then, Atiyah classes have been interpreted and used extensively in different contexts, for instance, in studying Kähler and hyper-Kähler manifolds and Hochschild cohomology (see, e.g., [15], [20], and [11]).

Our main result (see Theorem 6.10 for the complete statement) is a vanishing theorem (analogous to the Bott vanishing theorem) for Atiyah forms in the presence of not necessarily involutive holomorphic distributions:

Theorem 0.1. Let $M$ be a complex manifold of dimension $n$ and $F$ a holomorphic subbundle of rank $r$ of $T M$. Let $E$ be a holomorphic vector bundle over $M$ and $(F, \delta)$ a holomorphic partial connection for $E$. If $\nabla$ is a connection on E extending $\delta$, then

$$
a^{d}(\nabla) \equiv 0
$$

for all $d>n-r$, where $a^{d}(\nabla)$ denotes the Atiyah form of bidegree $(d, d)$. 
This result provides the foundation for the residue theory of singular (and not necessarily involutive) distributions that we shall describe in Section 8. As an application, we present a Camacho-Sad type result (Theorem 7.1); and we shall also work out an explicit example (Section 9).

To obtain these results, we exploit a presentation of Atiyah forms and classes based on a Chern-Weil type construction via $C^{\infty}$ connections of type $(1,0)$ and expressed by using Cech-Dolbeault cohomology. This viewpoint is particularly suited for developing the localization theory of characteristic classes; furthermore it allows us to understand and formulate clearly the relations between Chern and Atiyah classes, because (see Section 1.3) we may compare directly the differential forms representing Atiyah classes with those representing Chern classes, whereas this would not be possible in general at the level of classes because Chern classes and Atiyah classes live in different cohomologies.

The use of $(1,0)$-connections in this setting was already present in [4] in the framework of principal bundles; and the idea of incorporating this into the ChernWeil theory had been noted in [5]. We bring these ideas to fruition by combining them with the Cech-Dolbeault cohomology in order to suitably localize Atiyah classes. We note that here the difference forms play a key role. These tactics are analogues of those for Chern classes where the Chern-Weil theory is combined with the Čech-de Rham cohomology (originally due to D. Lehmann [16], see also [23]).

The plan of the paper is the following. In Section 1 we describe Atiyah classes using connections of type $(1,0)$, as said above; the comparison with the original definition is carried out in Section 2. In Section 3 we summarize the Čech-Dolbeault cohomology theory, so that in Section 4 we can express Atiyah classes in the CechDolbeault cohomology and explain the basic principle of localization; a vanishing theorem always yields a corresponding localization theorem with the associated residues. In Section 5 we briefly discuss the localization by sections, or more generally, by frames. In Section 6 we prove our main theorem, a Bott type vanishing theorem for nonsingular distributions, the starting point for the residue theory of singular distributions that we shall discuss in Section 8. As an important example, we give the vanishing theorem coming from the Camacho-Sad action in Section 7. Finally, in Section 9 we compute the Atiyah residues for an example of a singular distribution.

\section{Atiyah classes}

For details of the Chern-Weil theory of characteristic classes of complex vector bundles, we refer to [6], [7], [21], and [23]. Here we use the notation in [23] (with connection and curvature matrices transposed and $r$ and $\ell$ interchanged).

\subsection{Atiyah forms}

Let $M$ be a complex manifold and $E$ a holomorphic vector bundle over $M$ of rank $\ell$. For an open set $U$ in $M$, we denote by $A^{p}(U)$ the complex vector space of complex valued $C^{\infty} p$-forms on $U$. Also, we let $A^{p}(U, E)$ be the vector space of " $E$-valued $p$-forms" on $U$, that is, $C^{\infty}$ sections of the bundle $\bigwedge^{p}\left(T_{\mathbb{R}}^{c} M\right)^{*} \otimes E$ on $U$, 
where $\left(T_{\mathbb{R}}^{c} M\right)^{*}$ denotes the dual of the complexification of the real tangent bundle $T_{\mathbb{R}} M$ of $M$. Thus $A^{0}(U)$ is the ring of $C^{\infty}$ functions and $A^{0}(U, E)$ is the $A^{0}(U)$ module of $C^{\infty}$ sections of $E$ on $U$.

Definition 1.1. A $\left(C^{\infty}\right)$ connection for $E$ is a $\mathbb{C}$-linear map

$$
\nabla: A^{0}(M, E) \longrightarrow A^{1}(M, E)
$$

satisfying the Leibniz rule

$$
\nabla(f s)=d f \otimes s+f \nabla(s) \quad \text { for } \quad f \in A^{0}(M) \text { and } s \in A^{0}(M, E) .
$$

Definition 1.2. For $r=1, \ldots, \ell$, an $r$-frame of $E$ on an open set $U$ is a collection $s^{(r)}=\left(s_{1}, \ldots, s_{r}\right)$ of $r$ sections of $E$ linearly independent everywhere on $U$. An $\ell$-frame is simply called a frame.

Definition 1.3. Let $\nabla$ be a connection for $E$ on $U$, and $s^{(r)}=\left(s_{1}, \ldots, s_{r}\right)$ an $r$-frame of $E$. We say that $\nabla$ is $s^{(r)}$-trivial if $\nabla\left(s_{i}\right)=0$ for $i=1, \ldots, r$.

A connection $\nabla$ for $E$ induces a $\mathbb{C}$-linear map

$$
\nabla: A^{1}(M, E) \longrightarrow A^{2}(M, E)
$$

satisfying

$$
\nabla(\omega \otimes s)=d \omega \otimes s-\omega \wedge \nabla(s) \quad \text { for } \quad \omega \in A^{1}(M) \text { and } s \in A^{0}(M, E) .
$$

The composition

$$
K=\nabla \circ \nabla: A^{0}(M, E) \longrightarrow A^{2}(M, E)
$$

is called the curvature of $\nabla$. It is not difficult to see that $K$ is $A^{0}(M)$-linear; hence it can be thought of as a $C^{\infty} 2$-form with coefficients in the bundle $\operatorname{Hom}(E, E)$.

Notice that a connection is a local operator, i.e., it is also defined on local sections. This fact allows us to obtain local representations of a connection and its curvature by matrices whose entries are differential forms. Thus suppose that $\nabla$ is a connection for $E$. If $e^{(\ell)}=\left(e_{1}, \ldots, e_{\ell}\right)$ is a frame of $E$ on $U$, we may write, for $i=1, \ldots, \ell$,

$$
\nabla\left(e_{i}\right)=\sum_{j=1}^{\ell} \theta_{i}^{j} \otimes e_{j} \quad \text { with } \theta_{i}^{j} \text { in } A^{1}(U) .
$$

The matrix $\theta=\left(\theta_{i}^{j}\right)$ is the connection matrix of $\nabla$ with respect to $e^{(\ell)}$. Also, from the definition we get

$$
K\left(e_{i}\right)=\sum_{j=1}^{\ell} \kappa_{i}^{j} \otimes e_{j} \quad \text { with } \quad \kappa_{i}^{j}=d \theta_{i}^{j}+\sum_{k=1}^{\ell} \theta_{k}^{j} \wedge \theta_{i}^{k} .
$$

We call $\kappa=\left(\kappa_{i}^{j}\right)$ the curvature matrix of $\nabla$ with respect to $e^{(\ell)}$. 
If $\tilde{e}^{(\ell)}=\left(\tilde{e}_{1}, \ldots, \tilde{e}_{\ell}\right)$ is another frame of $E$ on $\tilde{U}$, we have $\tilde{e}_{i}=\sum_{j=1}^{\ell} a_{i}^{j} e_{j}$ for suitable functions $a_{i}^{j} \in A^{0}(U \cap \tilde{U})$, and the matrix $A=\left(a_{i}^{j}\right)$ is nonsingular at each point of $U \cap \tilde{U}$. If we denote by $\tilde{\theta}$ and $\tilde{\kappa}$ the connection and curvature matrices of $\nabla$ with respect to $\tilde{e}^{(\ell)}$ we have

$$
\tilde{\theta}=A^{-1} \cdot d A+A^{-1} \theta A \text { and } \tilde{\kappa}=A^{-1} \kappa A \text { in } U \cap \tilde{U} .
$$

Up to now $E$ could have been only a $C^{\infty}$ complex vector bundle. Now we use the assumption that $E$ is holomorphic.

Definition 1.4. A connection $\nabla$ for $E$ is of type $(1,0)$ (or a $(1,0)$-connection) if the entries of the connection matrix with respect to a holomorphic frame are forms of type $(1,0)$.

Remark 1.5. 1) It is easy to check that the above property does not depend on the choice of the holomorphic frame.

2) A holomorphic vector bundle always admits a $(1,0)$-connection. In fact let $\mathcal{V}=\left\{V_{\lambda}\right\}$ be an open covering of $M$ trivializing $E$. For each $\lambda$, let $\nabla_{\lambda}$ be the connection trivial with respect to some holomorphic frame on $V_{\lambda}$. If we take a partition of unity $\left\{\rho_{\lambda}\right\}$ subordinate to $\mathcal{V}$ and set $\nabla=\sum_{\lambda} \rho_{\lambda} \nabla_{\lambda}$, then $\nabla$ is a (1,0)-connection for $E$.

If $\nabla$ is a $(1,0)$-connection for $E$, we may write its curvature $K$ as

$$
K=K^{2,0}+K^{1,1}
$$

with $K^{2,0}$ and $K^{1,1}$, respectively, a $(2,0)$-form and a $(1,1)$-form with coefficients in $\operatorname{Hom}(E, E)$. Locally, if $\theta$ and $\kappa$ are respectively the connection and the curvature matrices of $\nabla$ with respect to a (local) holomorphic frame of $E$, then we can decompose $\kappa=\kappa^{2,0}+\kappa^{1,1}$ according to type, and $K^{2,0}$ and $K^{1,1}$ are respectively represented by

$$
\kappa^{2,0}=\partial \theta+\theta \wedge \theta \quad \text { and } \quad \kappa^{1,1}=\bar{\partial} \theta .
$$

Thus $K^{1,1}$, being locally $\bar{\partial}$-exact, is a $\bar{\partial}$-closed $(1,1)$-form with coefficients in $\operatorname{Hom}(E, E)$.

With respect to another holomorphic frame, $K^{1,1}$ is represented by a matrix similar to $\kappa^{1,1}$ (cf. (1.1)). Thus for each elementary symmetric polynomial $\sigma_{p}$ (with $p=1,2, \ldots)$ we may define a $\bar{\partial}$-closed $C^{\infty}(p, p)$-form $\sigma_{p}\left(K^{1,1}\right)$ on $M$. Locally it is given by $\sigma_{p}\left(\kappa^{1,1}\right)$, which is the coefficient of $t^{p}$ in the expansion

$$
\operatorname{det}\left(I+t \kappa^{1,1}\right)=1+\sigma_{1}\left(\kappa^{1,1}\right) t+\cdots+\sigma_{p}\left(\kappa^{1,1}\right) t^{p}+\cdots .
$$

In particular, $\sigma_{1}\left(\kappa^{1,1}\right)=\operatorname{tr}\left(\kappa^{1,1}\right)$ and $\sigma_{\ell}\left(\kappa^{1,1}\right)=\operatorname{det}\left(\kappa^{1,1}\right)$.

Definition 1.6. Let $\nabla$ be a $(1,0)$-connection for a holomorphic vector bundle $E$ of rank $\ell$. For $p=1, \ldots, \ell$, we define the $p$-th Atiyah form $a^{p}(\nabla)$ of $\nabla$ by

$$
a^{p}(\nabla)=\left(\frac{\sqrt{-1}}{2 \pi}\right)^{p} \sigma_{p}\left(K^{1,1}\right) .
$$

It is a $\bar{\partial}$-closed $(p, p)$-form on $M$. 
More generally, if $\varphi$ is a symmetric homogeneous polynomial of degree $d$, we may write $\varphi=P\left(\sigma_{1}, \sigma_{2}, \ldots\right)$ for a suitable polynomial $P$. Then we define the Atiyah form $\varphi^{A}(\nabla)$ of $\nabla$ associated to $\varphi$ by

$$
\varphi^{A}(\nabla)=P\left(a^{1}(\nabla), a^{2}(\nabla), \ldots\right)
$$

it is a $\bar{\partial}$-closed $(d, d)$-form on $M$.

Remark 1.7. The $p$-th Chern form $c^{p}(\nabla)$ of $\nabla$ is defined by

$$
c^{p}(\nabla)=\left(\frac{\sqrt{-1}}{2 \pi}\right)^{p} \sigma_{p}(\kappa),
$$

which is a closed $(2 p)$-form having components of bidegrees $(2 p, 0), \ldots,(p, p)$. The Atiyah form $a^{p}(\nabla)$ is then the $(p, p)$-component of $c^{p}(\nabla)$. In particular, $a^{n}(\nabla)=$ $c^{n}(\nabla)$, where $n$ denotes the dimension of $M$.

More generally, the Atiyah form $\varphi^{A}(\nabla)$ of $\nabla$ associated to a symmetric homogeneous polynomial $\varphi$ of degree $d$ is the component of type $(d, d)$ of the Chern form $\varphi(\nabla)=P\left(c^{1}(\nabla), c^{2}(\nabla), \ldots\right)$ associated to $\varphi$. Again, if $d=n$ then $\varphi^{A}(\nabla)=\varphi(\nabla)$.

\subsection{Atiyah classes}

Let $E$ be a holomorphic vector bundle over a complex manifold $M$. As in the case of Chern forms, to any finite set of $(1,0)$-connections for $E$ one can associate a difference form. Here we recall the construction given in Proposition 5.4 of [25].

Thus, given $q+1(1,0)$-connections $\nabla_{0}, \ldots, \nabla_{q}$ for $E$, we consider the vector bundle $E \times \mathbb{R}^{q} \rightarrow M \times \mathbb{R}^{q}$ and define the connection $\tilde{\nabla}$ for the bundle by $\tilde{\nabla}=$ $\left(1-\sum_{i=1}^{q} t_{i}\right) \nabla_{0}+\sum_{i=1}^{q} t_{i} \nabla_{i}$, where $\left(t_{1} \ldots, t_{q}\right)$ denote coordinates on $\mathbb{R}^{q}$. Denoting by $\Delta^{q}$ the standard $q$-simplex in $\mathbb{R}^{q}$ and by $\pi: M \times \Delta^{q} \rightarrow M$ the projection, we have the integration along the fiber

$$
\pi_{*}: A^{2 p}\left(M \times \Delta^{q}\right) \longrightarrow A^{2 p-q}(M) .
$$

Then we set

$$
c^{p}\left(\nabla_{0}, \ldots, \nabla_{q}\right):=\pi_{*}\left(c^{p}(\tilde{\nabla})\right) .
$$

The Atiyah difference form $a^{p}\left(\nabla_{0}, \ldots, \nabla_{q}\right)$ is the $(p, p-q)$-component of the form $c^{p}\left(\nabla_{0}, \ldots, \nabla_{q}\right)$. It is alternating in the $q+1$ entries and satisfies

$$
\sum_{\nu=0}^{q} a^{p}\left(\nabla_{0}, \ldots, \widehat{\nabla}_{\nu}, \ldots, \nabla_{q}\right)+(-1)^{q} \bar{\partial} a^{p}\left(\nabla_{0}, \ldots, \nabla_{q}\right)=0 .
$$

In particular, if $q=1$, we have

$$
\bar{\partial} a^{p}\left(\nabla_{0}, \nabla_{1}\right)=a^{p}\left(\nabla_{1}\right)-a^{p}\left(\nabla_{0}\right),
$$

which shows that, if $\nabla$ is a $(1,0)$-connection for $E$, the class of $a^{p}(\nabla)$ in $H_{\bar{\partial}}^{p, p}(M)$ does not depend on the choice of $\nabla$.

Similarly, if $\varphi$ is a symmetric homogeneous polynomial of degree $d$, we can define a $(d, d-q)$-form $\varphi^{A}\left(\nabla_{0}, \ldots, \nabla_{q}\right)$ as the $(d, d-q)$-component of the Chern difference form $\varphi\left(\nabla_{0}, \ldots, \nabla_{q}\right)$. It satisfies an identity analogous to (1.3). 
Then we can introduce the following definition:

Definition 1.8. Let $E$ be a holomorphic vector bundle $E$ of rank $\ell$. For $p=$ $1, \ldots, \ell$ the $p$-th Atiyah class $a^{p}(E)$ of $E$ is the class represented by $a^{p}(\nabla)$ in $H_{\bar{\partial}}^{p, p}(M)$, where $\nabla$ is an arbitrary $(1,0)$-connection for $E$.

Similarly, if $\varphi$ is a symmetric homogeneous polynomial of degree $d$, the Atiyah class $\varphi^{A}(E)$ of $E$ associated to $\varphi$ is the class of $\varphi^{A}(\nabla)$ in $H_{\bar{\partial}}^{d, d}(M)$, where $\nabla$ is an arbitrary $(1,0)$-connection for $E$.

Remark 1.9. If $n$ denotes the dimension of $M$, there is a canonical surjective map $H_{\bar{\partial}}^{n, n}(M) \rightarrow H_{\mathrm{dR}}^{2 n}(M)$, the de Rham cohomology of $M$, which assigns the class of a form $\omega$ to the class of $\omega$. If $d=n$, then $\varphi^{A}(\nabla)=\varphi(\nabla)$ for any $(1,0)$-connection $\nabla$ for $E$ and the image of $\varphi^{A}(E)$ by the above map is $\varphi(E)$. In particular, if $M$ is compact, $\int_{M} \varphi^{A}(E)=\int_{M} \varphi(E)$.

Moreover, if $d=n$, then $\varphi^{A}\left(\nabla_{0}, \nabla_{1}\right)$ also coincides with the usual Bott difference form $\varphi\left(\nabla_{0}, \nabla_{1}\right)$ for any pair $\nabla_{0}, \nabla_{1}$ of $(1,0)$-connections for $E$.

\subsection{Atiyah classes on compact Kähler manifolds}

Let $M$ be a complex manifold (not necessarily Kähler) and $E$ a holomorphic vector bundle on $M$. Let $h$ be any Hermitian metric on $E$ and let $\nabla^{h}$ be the associated metric connection, i.e., $\nabla^{h}$ is the unique $(1,0)$-connection compatible with $h$. The curvature $K$ of $\nabla$ is then of type $(1,1)$, and hence

$$
c^{p}\left(\nabla^{h}\right)=a^{p}\left(\nabla^{h}\right) \quad \text { for all } p \geq 1 .
$$

In other words, Atiyah and Chern classes of the same degree can be represented by the same form. Of course, as classes they are different, because they belong to two different cohomology groups: $c^{p}(E)=\left[c^{p}\left(\nabla^{h}\right)\right] \in H_{\mathrm{dR}}^{2 p}(M)$, while $a^{p}(E)=$ $\left[a^{p}\left(\nabla^{h}\right)\right] \in H_{\bar{\partial}}^{p, p}(M)$, the Dolbeault cohomology of $M$.

However, if $M$ is compact Kähler, the Hodge decomposition yields a canonical injection $H_{\bar{\partial}}^{p, p}(M) \hookrightarrow H_{\mathrm{dR}}^{2 p}(M)$, and hence we obtain the following useful relation:

Proposition 1.10. Let $M$ be a compact Kähler manifold and $E$ a holomorphic vector bundle on $M$. Let $I: H_{\bar{\partial}}^{p, p}(M) \rightarrow H_{\mathrm{dR}}^{2 p}(M)$ be the injection given by the Hodge decomposition. Then

$$
I\left(a^{p}(E)\right)=c^{p}(E) \quad \text { for all } p \geq 1 .
$$

\section{Atiyah classes via complex analytic connections}

Atiyah classes were originally introduced by Atiyah in [4], with a different construction. In this section we show that our definition yields the same classes.

Let $M$ be a complex manifold and $\mathcal{O}$ the sheaf of germs of holomorphic functions on $M$. For a holomorphic vector bundle $E$ over $M$ we denote by $\mathcal{E}=\mathcal{O}(E)$ the sheaf of germs of holomorphic sections of $E$. We also denote by $\Theta=\mathcal{O}(T M)$ and $\Omega^{1}=\mathcal{O}\left(T^{*} M\right)$ the sheaves of germs holomorphic vector fields and of 1 -forms on $M$. All tensor products in this section will be over the sheaf $\mathcal{O}$. 
Definition 2.1. A holomorphic (or complex analytic) connection for $E$ is a homomorphism of sheaves of $\mathbb{C}$-vector spaces

$$
\nabla: \mathcal{E} \longrightarrow \Omega^{1} \otimes \mathcal{E}
$$

satisfying

$$
\nabla(f s)=d f \otimes s+f \nabla(s) \quad \text { for } f \in \mathcal{O} \text { and } s \in \mathcal{E} .
$$

If $e^{(r)}=\left(e_{1}, \ldots, e_{r}\right)$ is a local holomorphic $r$-frame of $E$, we shall say that $\nabla$ is $e^{(r)}$-trivial if $\nabla e_{j} \equiv 0$ for $j=1, \ldots, r$.

Remark 2.2. A holomorphic connection $\boldsymbol{\nabla}$ on a holomorphic vector bundle $E$ induces naturally a $(1,0)$-connection $\nabla$. In fact, let $s$ be a $C^{\infty}$ section of $E$. Let $U$ be an open set trivializing $E$ and let $\left(e_{1}, \ldots, e_{\ell}\right)$ be a holomorphic frame on $U$. Write $s=\sum_{i=1}^{\ell} f^{i} e_{i}$ with $f^{i} \in C^{\infty}(U)$, and set $\nabla s=\sum_{i=1}^{\ell}\left(d f^{i} \otimes e_{i}+f^{i} \nabla\left(e_{i}\right)\right)$. It is easy to check that the definition does not depend on the choice of the frame.

Conversely, a $(1,0)$-connection $\nabla$ such that $(\nabla s)(u)$ is holomorphic wherever $s$ and $u$ are holomorphic clearly determines a holomorphic connection.

Following Atiyah [4], we set

$$
D(\mathcal{E}):=\mathcal{E} \oplus\left(\Omega^{1} \otimes \mathcal{E}\right),
$$

which is a direct sum as a sheaf of $\mathbb{C}$-vector spaces. It is endowed with the $\mathcal{O}$-module structure given by

$$
f \cdot(s, \sigma)=(f s, d f \otimes s+f \sigma) .
$$

Then we have the following exact sequence of (locally free) $\mathcal{O}$-modules:

$$
0 \longrightarrow \Omega^{1} \otimes \mathcal{E} \stackrel{\iota}{\longrightarrow} D(\mathcal{E}) \stackrel{\rho}{\longrightarrow} \mathcal{E} \longrightarrow 0 .
$$

A splitting of this sequence is a morphism $\eta: \mathcal{E} \rightarrow D(\mathcal{E})$ of $\mathcal{O}$-modules such that $\rho \circ \eta=$ id.

Lemma 2.3 ([4]). Let $E$ be a holomorphic vector bundle on a complex manifold $M . A$ morphism $\eta: \mathcal{E} \rightarrow D(\mathcal{E})$ is a splitting of (2.1) if and only if it is of the form $\eta(s)=(s, \nabla(s))$, where $\nabla$ is a holomorphic connection for $E$. Thus $E$ admits a holomorphic connection if and only if (2.1) splits.

The following is also easy to see:

Lemma 2.4 ([4]). Let $\boldsymbol{\nabla}$ be a holomorphic connection for a holomorphic vector bundle $E$. If $\xi \in \mathcal{H o m}_{\mathcal{O}}\left(\mathcal{E}, \Omega^{1} \otimes \mathcal{E}\right)$ then $\nabla+\xi$ is a holomorphic connection for $E$. Conversely, every holomorphic connection for $E$ is of this form.

We see that the sequence $(2.1)$ determines an element $b(E)$ in the cohomology $H^{1}\left(M, \mathcal{H o m}\left(\mathcal{E}, \Omega^{1} \otimes \mathcal{E}\right)\right)$ as follows. First, applying the functor $\mathcal{H o m}(\mathcal{E}, \cdot)$ to $(2.1)$ we get the exact sequence

$$
0 \longrightarrow \mathcal{H o m}\left(\mathcal{E}, \Omega^{1} \otimes \mathcal{E}\right) \longrightarrow \mathcal{H o m}(\mathcal{E}, D(\mathcal{E})) \longrightarrow \mathcal{H o m}(\mathcal{E}, \mathcal{E}) \longrightarrow 0
$$


and thus the connecting homomorphism

$$
\delta: H^{0}(M, \mathcal{H o m}(\mathcal{E}, \mathcal{E})) \longrightarrow H^{1}\left(M, \mathcal{H o m}\left(\mathcal{E}, \Omega^{1} \otimes \mathcal{E}\right)\right)
$$

Then $b(E)=\delta($ id $) \in H^{1}\left(M, \mathcal{H o m}\left(\mathcal{E}, \Omega^{1} \otimes \mathcal{E}\right)\right)$. It is not difficult to prove the following:

Lemma 2.5 ([4]). A holomorphic vector bundle E admits a holomorphic connection if and only if $b(E)=0$.

Now, we have the Dolbeault isomorphism

$$
H^{1}\left(M, \mathcal{H o m}\left(\mathcal{E}, \Omega^{1} \otimes \mathcal{E}\right)\right)=H^{1}\left(M, \Omega^{1} \otimes \mathcal{H o m}(\mathcal{E}, \mathcal{E})\right) \simeq H_{\bar{\partial}}^{1,1}(M, \operatorname{Hom}(E, E)) .
$$

Let $a(E)$ denote the class in $H_{\bar{\partial}}^{1,1}(M, \operatorname{Hom}(E, E))$ corresponding to $-b(E)$ via the above isomorphism (cf. Theorem 5 in [4]). Then the original Atiyah class of type $(p, p)$ is defined as

$$
a_{\mathrm{or}}^{p}(E)=\left(\frac{\sqrt{-1}}{2 \pi}\right)^{p} \sigma_{p}(a(E))
$$

we shall show that $a_{\mathrm{or}}^{p}(E)=a^{p}(E)$ for all $p \geq 1$. To do so we need some definitions.

Definition 2.6. Let $\mathcal{V}=\left\{V_{\lambda}\right\}$ be an open covering of $M$. A $\mathcal{V}$-splitting of (2.1) is a collection $\left\{\eta^{\lambda}\right\}$ of splittings $\eta^{\lambda}$ of (2.1) on each $V_{\lambda}$. A holomorphic $\mathcal{V}$-connection for $E$ is a collection $\left\{\nabla^{\lambda}\right\}$ of holomorphic connections $\nabla^{\lambda}$ for $\left.E\right|_{V_{\lambda}}$.

By Lemma 2.3, a $\mathcal{V}$-splitting determines a holomorphic $\mathcal{V}$-connection and viceversa. Furthermore, every holomorphic vector bundle $E$ admits a holomorphic $\mathcal{V}$-connection for some open covering $\mathcal{V}$. In fact, let $\mathcal{V}=\left\{V_{\lambda}\right\}$ be a covering trivializing $E$; then take as $\nabla^{\lambda}$ a holomorphic connection which is trivial with respect to some holomorphic frame of $E$ on $V_{\lambda}$.

Definition 2.7. We shall call $\bar{\partial}$-curvature of $E$ a $\bar{\partial}$-closed $(1,1)$-form with coefficients in $\operatorname{Hom}(E, E)$ representing the class $a(E)$.

The next theorem shows that we can get a $\bar{\partial}$-curvature as the $(1,1)$-component of the curvature of a suitable $(1,0)$-connection:

Theorem 2.8. Let $E$ be a holomorphic vector bundle over a complex manifold $M$. $A$ holomorphic $\mathcal{V}$-connection for $E$ determines a $(1,0)$-connection $\nabla$ for $E$ such that the $(1,1)$-component of the curvature of $\nabla$ is a $\bar{\partial}$-curvature.

Proof. Let $\left\{\nabla^{\lambda}\right\}$ be a $\mathcal{V}$-connection for $E$ with respect to a (sufficiently fine) open covering $\mathcal{V}=\left\{V_{\lambda}\right\}$ of $M$. On $V_{\lambda} \cap V_{\mu}$ the difference $\xi^{\lambda \mu}=\nabla^{\lambda}-\nabla^{\mu}$ is an $\mathcal{O}$-morphism from $\mathcal{E}$ to $\Omega^{1} \otimes \mathcal{E}$, and the collection $\xi=\left\{\xi^{\lambda \mu}\right\}$ is a 1-cocycle on $\mathcal{V}$ representing $-b(E)$.

We denote by $\mathcal{A}^{p, q}(\operatorname{Hom}(E, E))$ the sheaf of germs of smooth forms of type $(p, q)$ with coefficients in the bundle $\operatorname{Hom}(E, E)$; in particular, we may think of 
$\mathcal{H o m}\left(\mathcal{E}, \Omega^{1} \otimes \mathcal{E}\right)=\Omega^{1} \otimes \mathcal{H o m}(\mathcal{E}, \mathcal{E})$ as a subsheaf of $\mathcal{A}^{1,0}(\operatorname{Hom}(E, E))$. Since the sheaf $\mathcal{A}^{p, q}(\operatorname{Hom}(E, E))$ is fine, there exists a 0 -cochain $\left\{\tau^{\lambda}\right\}$ of $\mathcal{A}^{1,0}(\operatorname{Hom}(E, E))$ on $\mathcal{V}$ such that

$$
\xi^{\lambda \mu}=\tau^{\mu}-\tau^{\lambda} \quad \text { on } V_{\lambda} \cap V_{\mu} .
$$

Hence

$$
\nabla^{\lambda}+\tau^{\lambda}=\nabla^{\mu}+\tau^{\mu} \quad \text { on } V_{\lambda} \cap V_{\mu} .
$$

In this way we have defined a global $(1,0)$-connection $\nabla$ which coincides with $\nabla^{\lambda}+\tau^{\lambda}$ on $V_{\lambda}$.

Since the forms $\xi^{\lambda \mu}$ are holomorphic, on $V_{\lambda} \cap V_{\mu}$ we have $\bar{\partial} \tau^{\lambda}=\bar{\partial} \tau^{\mu}$. Hence we get a global $\bar{\partial}$-closed $(1,1)$-form $\omega$ with coefficients in $\operatorname{Hom}(E, E)$ which is equal to $\bar{\partial} \tau^{\lambda}$ on $V_{\lambda}$. By chasing diagrams, it is easy to see that the form $\omega$ represents the class $a(E)$, and thus it is a $\bar{\partial}$-curvature. Moreover, (1.2) shows that $\omega$ is the $(1,1)$-component of the curvature of $\nabla$, and we are done.

Corollary 2.9. Let $E$ be a holomorphic vector bundle on a complex manifold $M$. Then

$$
a_{\mathrm{or}}^{p}(E)=a^{p}(E)
$$

for all $p \geq 1$.

Remark 2.10. Given a holomorphic $\mathcal{V}$-connection $\left\{\nabla^{\lambda}\right\}$, the $\bar{\partial}$-curvature $\omega$ constructed in the proof of Theorem 2.8 is not uniquely determined; it depends on the choice of the 0 -cochain $\left\{\tau^{\lambda}\right\}$. One way to choose $\left\{\tau^{\lambda}\right\}$ is to take a partition of unity $\left\{\rho_{\lambda}\right\}$ subordinate to $\mathcal{V}$ and set $\tau^{\lambda}=\sum_{\nu} \rho_{\nu} \xi^{\nu \lambda}$.

We give now a more explicit expression for the forms introduced in the proof of Theorem 2.8. Let $\ell$ be the rank of $E$, and choose an open cover $\mathcal{V}$ of sufficiently small open sets trivializing $E$. On each $V_{\lambda}$ take a holomorphic frame $\left(e_{1}^{\lambda}, \ldots, e_{\ell}^{\lambda}\right)$ of $E$ and let $\nabla^{\lambda}$ be the connection on $V_{\lambda}$ trivial with respect to this frame. Finally, let $\left\{h^{\lambda \mu}\right\}$ be the system of transition matrices corresponding to these choices, that is

$$
e_{j}^{\mu}=\sum_{k=1}^{\ell}\left(h^{\lambda \mu}\right)_{j}^{k} e_{k}^{\lambda} \quad \text { on } V_{\lambda} \cap V_{\mu} .
$$

Then

$$
\xi^{\lambda \mu}\left(e_{i}^{\lambda}\right)=-\sum_{j, k=1}^{\ell}\left(h^{\lambda \mu}\right)_{k}^{j} \cdot d\left(h^{\mu \lambda}\right)_{i}^{k} \otimes e_{j}^{\lambda} .
$$

Thus, with respect to the frame $\left(e_{1}^{\lambda}, \ldots, e_{\ell}^{\lambda}\right), \xi^{\lambda \mu}$ is represented, as an element of $\mathcal{H o m}\left(\mathcal{E}, \Omega^{1} \otimes \mathcal{E}\right) \simeq \Omega^{1} \otimes \mathcal{H o m}(\mathcal{E}, \mathcal{E})$ on $V_{\lambda} \cap V_{\mu}$, by the matrix

$$
-h^{\lambda \mu} \cdot d h^{\mu \lambda}=d h^{\lambda \mu} \cdot\left(h^{\lambda \mu}\right)^{-1}=\partial h^{\lambda \mu} \cdot\left(h^{\lambda \mu}\right)^{-1} .
$$

Taking a partition of unity $\left\{\rho_{\lambda}\right\}$ subordinate to $\mathcal{V}$, we may set $\tau^{\lambda}=\sum_{\nu} \rho_{\nu} \xi^{\nu \lambda}$, as in Remark 2.10; the global $(1,0)$-connection constructed in the proof of Theorem 2.8 is then given by $\nabla=\sum_{\nu} \rho_{\nu} \nabla^{\nu}$, and its curvature matrix with respect to the frame $\left(e_{1}^{\lambda}, \ldots, e_{\ell}^{\lambda}\right)$ is given by $\tau^{\lambda}$, and the corresponding $\bar{\partial}$-curvature by $\bar{\partial} \tau^{\lambda}$. As a direct consequence of Lemma 2.5 and Corollary 2.9 we get: 
Proposition 2.11. Let $E$ be a holomorphic vector bundle over a complex manifold $M$. If $E$ admits a holomorphic connection then $a^{p}(E)=0$ for all $p \geq 1$, that is, all Atiyah classes of E vanish.

Remark 2.12. In fact, the existence of a holomorphic connection $\nabla$ implies the stronger vanishing $a^{p}(\nabla)=0$ for all $p \geq 1$. This can be easily seen from (1.2), since the connection matrix $\theta$ of $\nabla$ with respect to a holomorphic frame is holomorphic. See Theorem 6.10 below for more general vanishing results of this type.

It should be remarked that the converse of Proposition 2.11 is not true. Namely, it might happen that $a^{p}(E)=0$ for all $p \geq 1$ but $a(E) \neq 0$, as the following example shows.

Example 2.13. Let $M$ be a compact Riemann surface and $L$ a line bundle over $M$ such that $a^{1}(L)=c^{1}(L) \neq 0$. Let $E:=L \oplus L^{*}$. Then $c^{1}(E)=c^{1}(L)-c^{1}(L)=0$, and by Proposition 1.10 it follows $a^{1}(E)=0$. For dimensional reasons, $a^{p}(E)=0$ for all $p \geq 2$. Now we claim that $E$ does not admit a holomorphic connection, and hence $a(E) \neq 0$ as a class in $H_{\bar{\partial}}^{1,1}(M, \operatorname{Hom}(E, E))$. In fact, aiming at a contradiction, let $\nabla$ denote a holomorphic connection for $E$. Let $\pi: \Omega^{1} \otimes E \rightarrow \Omega^{1} \otimes L$ denote the projection and $\iota: L \rightarrow E$ the immersion. It is easy to show that $\pi \circ \nabla \circ \iota$ is a holomorphic connection for $L$. But then $c^{1}(L)=a^{1}(L)=0$, contrary to our assumption.

\section{3. Čech-Dolbeault cohomology}

In this section, we recall the theory of Čech-Dolbeault cohomology in the case of coverings consisting of two open sets. Although it is technically more involved, the ideas are similar for the general case of coverings with arbitrary number of open sets. We review relevant material for this case in Section 9 and refer to [25] for details

Let $M$ be a complex manifold of dimension $n$. For an open set $U$ of $M$, we denote by $A^{p, q}(U)$ the vector space of $C^{\infty}(p, q)$-forms on $U$. Let $\mathcal{U}=\left\{U_{0}, U_{1}\right\}$ be an open covering of $M$. Set $U_{01}=U_{0} \cap U_{1}$ and define the vector space $A^{p, q}(\mathcal{U})$ by

$$
A^{p, q}(\mathcal{U})=A^{p, q}\left(U_{0}\right) \oplus A^{p, q}\left(U_{1}\right) \oplus A^{p, q-1}\left(U_{01}\right) .
$$

Thus an element $\sigma$ in $A^{p, q}(\mathcal{U})$ is given by a triple $\sigma=\left(\sigma_{0}, \sigma_{1}, \sigma_{01}\right)$ with $\sigma_{i}$ a $(p, q)$-form on $U_{i}, i=0,1$, and $\sigma_{01}$ a $(p, q-1)$-form on $U_{01}$.

We define a differential operator $\bar{D}: A^{p, q}(\mathcal{U}) \rightarrow A^{p, q+1}(\mathcal{U})$ by

$$
\bar{D} \sigma=\left(\bar{\partial} \sigma_{0}, \bar{\partial} \sigma_{1}, \sigma_{1}-\sigma_{0}-\bar{\partial} \sigma_{01}\right) \text {. }
$$

Then we have $\bar{D} \circ \bar{D}=0$ and thus a complex for each fixed $p$ :

$$
\cdots \longrightarrow A^{p, q-1}(\mathcal{U}) \stackrel{\bar{D}^{p, q-1}}{\longrightarrow} A^{p, q}(\mathcal{U}) \stackrel{\bar{D}^{p, q}}{\longrightarrow} A^{p, q+1}(\mathcal{U}) \longrightarrow \cdots
$$


We set

$$
H_{\bar{D}}^{p, q}(\mathcal{U})=\operatorname{Ker} \bar{D}^{p, q} / \operatorname{Im} \bar{D}^{p, q-1}
$$

and call it the $\check{C}$ ech-Dolbeault cohomology of $\mathcal{U}$ of type $(p, q)$. We denote the image of $\sigma$ by the canonical surjection $\operatorname{Ker} \bar{D}^{p, q} \rightarrow H_{\bar{D}}^{p, q}(\mathcal{U})$ by $[\sigma]$.

Let $H_{\bar{\partial}}^{p, q}(M)$ denote the Dolbeault cohomology of $M$ of type $(p, q)$.

Theorem 3.1. The map $\alpha: A^{p, q}(M) \rightarrow A^{p, q}(\mathcal{U})$ given by $\omega \mapsto(\omega, \omega, 0)$ induces an isomorphism

$$
\alpha: H_{\bar{\partial}}^{p, q}(M) \stackrel{\sim}{\longrightarrow} H_{\bar{D}}^{p, q}(\mathcal{U}) .
$$

Proof. It is not difficult to show that $\alpha$ is well defined. To prove that $\alpha$ is surjective, let $\sigma=\left(\sigma_{0}, \sigma_{1}, \sigma_{01}\right)$ be such that $\bar{D} \sigma=0$. Let $\left\{\rho_{0}, \rho_{1}\right\}$ be a partition of unity subordinate to $\mathcal{U}$ and set $\omega=\rho_{0} \sigma_{0}+\rho_{1} \sigma_{1}-\bar{\partial} \rho_{0} \wedge \sigma_{01}$. Then it is easy to see that $\bar{\partial} \omega=0$ and $[(\omega, \omega, 0)]=[\sigma]$. The injectivity of $\alpha$ is also not difficult to show.

We define the cup product

$$
A^{p, q}(\mathcal{U}) \times A^{p^{\prime}, q^{\prime}}(\mathcal{U}) \longrightarrow A^{p+p^{\prime}, q+q^{\prime}}(\mathcal{U}),
$$

assigning to $\sigma$ in $A^{p, q}(\mathcal{U})$ and $\tau$ in $A^{p^{\prime}, q^{\prime}}(\mathcal{U})$ the element $\sigma \smile \tau$ in $A^{p+p^{\prime}, q+q^{\prime}}(\mathcal{U})$ given by

$$
\begin{aligned}
(\sigma \smile \tau)_{0} & =\sigma_{0} \wedge \tau_{0}, \quad(\sigma \smile \tau)_{1}=\sigma_{1} \wedge \tau_{1} \quad \text { and } \\
(\sigma \smile \tau)_{01} & =(-1)^{p+q} \sigma_{0} \wedge \tau_{01}+\sigma_{01} \wedge \tau_{1} .
\end{aligned}
$$

Then $\sigma \smile \tau$ is linear in $\sigma$ and $\tau$ and we have

$$
\bar{D}(\sigma \smile \tau)=\bar{D} \sigma \smile \tau+(-1)^{p+q} \sigma \smile \bar{D} \tau .
$$

Thus it induces the cup product

$$
H_{\bar{D}}^{p, q}(\mathcal{U}) \times H_{\bar{D}}^{p^{\prime}, q^{\prime}}(\mathcal{U}) \longrightarrow H_{\bar{D}}^{p+p^{\prime}, q+q^{\prime}}(\mathcal{U})
$$

compatible, via the isomorphism of Theorem 3.1, with the product in the Dolbeault cohomology induced from the exterior product of forms.

Now we recall the integration for the Cech-Dolbeault cohomology. Let $M$ and $\mathcal{U}=\left\{U_{0}, U_{1}\right\}$ be as above and let $\left\{R_{0}, R_{1}\right\}$ be a system of honeycomb cells adapted to $\mathcal{U}$ (cf. [16], [23]). Thus each $R_{i}, i=0,1$, is a real submanifold of dimension $2 n$ with $C^{\infty}$ boundary in $M$ such that $R_{i} \subset U_{i}, M=R_{0} \cup R_{1}$, and $\operatorname{Int} R_{0} \cap \operatorname{Int} R_{1}=\emptyset$. We set $R_{01}=R_{0} \cap R_{1}$, which is equal to $\partial R_{0}=-\partial R_{1}$ as an oriented manifold.

Suppose $M$ is compact; then each $R_{i}$ is compact and we may define the integration

$$
\int_{M}: A^{n, n}(\mathcal{U}) \longrightarrow \mathbb{C}
$$

as the sum

$$
\int_{M} \sigma=\int_{R_{0}} \sigma_{0}+\int_{R_{1}} \sigma_{1}+\int_{R_{01}} \sigma_{01}
$$


for $\sigma$ in $A^{n, n}(\mathcal{U})$. Then this induces an integration on cohomology

$$
\int_{M}: H_{\bar{D}}^{n, n}(\mathcal{U}) \longrightarrow \mathbb{C},
$$

which is compatible, via the isomorphism of Theorem 3.1, with the usual integration on the Dolbeault cohomology $H_{\bar{\partial}}^{n, n}(M)$. Also the bilinear pairing

$$
A^{p, q}(\mathcal{U}) \times A^{n-p, n-q}(\mathcal{U}) \longrightarrow A^{n, n}(\mathcal{U}) \longrightarrow \mathbb{C}
$$

defined as the composition of the cup product and the integration induces the Kodaira-Serre duality

$$
K S: H_{\bar{\partial}}^{p, q}(M) \simeq H_{\bar{D}}^{p, q}(\mathcal{U}) \stackrel{\sim}{\longrightarrow} H_{\bar{D}}^{n-p, n-q}(\mathcal{U})^{*} \simeq H_{\bar{\partial}}^{n-p, n-q}(M)^{*} .
$$

Now let $S$ be a closed set in $M$. Let $U_{0}=M \backslash S$ and $U_{1}$ a neighborhood of $S$ in $M$, and consider the covering $\mathcal{U}=\left\{U_{0}, U_{1}\right\}$ of $M$. We denote by $A^{p, q}\left(\mathcal{U}, U_{0}\right)$ the subspace of $A^{p, q}(\mathcal{U})$ consisting of elements $\sigma$ with $\sigma_{0}=0$, so that we have the exact sequence

$$
0 \longrightarrow A^{p, q}\left(\mathcal{U}, U_{0}\right) \longrightarrow A^{p, q}(\mathcal{U}) \longrightarrow A^{p, q}\left(U_{0}\right) \longrightarrow 0 .
$$

We see that the operator $\bar{D}$ maps $A^{p, q}\left(\mathcal{U}, U_{0}\right)$ into $A^{p, q+1}\left(\mathcal{U}, U_{0}\right)$. Denoting by $H_{\bar{D}}^{p, q}\left(\mathcal{U}, U_{0}\right)$ the $q$-th cohomology of the complex $\left(A^{p, *}\left(\mathcal{U}, U_{0}\right), \bar{D}\right)$, we have the long exact sequence

$$
\cdots \longrightarrow H_{\bar{\partial}}^{p, q-1}\left(U_{0}\right) \longrightarrow H_{\bar{D}}^{p, q}\left(\mathcal{U}, U_{0}\right) \longrightarrow H_{\bar{D}}^{p, q}(\mathcal{U}) \longrightarrow H_{\bar{\partial}}^{p, q}\left(U_{0}\right) \longrightarrow \cdots
$$

In view of the fact that $H_{\bar{D}}^{p, q}(\mathcal{U}) \simeq H_{\bar{\partial}}^{p, q}(M)$, we set

$$
H_{\bar{\partial}}^{p, q}(M, M \backslash S):=H_{\bar{D}}^{p, q}\left(\mathcal{U}, U_{0}\right) .
$$

Suppose $S$ is compact ( $M$ may not be) and let $\left\{R_{0}, R_{1}\right\}$ be a system of honeycomb cells adapted to $\mathcal{U}$. Then we may assume that $R_{1}$ is compact and we have the integration on $A^{n, n}\left(\mathcal{U}, U_{0}\right)$ given by

$$
\int_{M} \sigma=\int_{R_{1}} \sigma_{1}+\int_{R_{01}} \sigma_{01} .
$$

This again induces an integration on cohomology

$$
\int_{M}: H_{\bar{D}}^{n, n}\left(\mathcal{U}, U_{0}\right) \longrightarrow \mathbb{C} .
$$

The cup product (3.1) induces a pairing

$$
A^{p, q}\left(\mathcal{U}, U_{0}\right) \times A^{n-p, n-q}\left(U_{1}\right) \longrightarrow A^{n, n}\left(\mathcal{U}, U_{0}\right),
$$

which, followed by integration, gives a bilinear pairing

$$
A^{p, q}\left(\mathcal{U}, U_{0}\right) \times A^{n-p, n-q}\left(U_{1}\right) \longrightarrow \mathbb{C} .
$$

This induces a homomorphism

$$
\bar{A}: H_{\bar{\partial}}^{p, q}(M, M \backslash S)=H_{\bar{D}}^{p, q}\left(\mathcal{U}, U_{0}\right) \longrightarrow H_{\bar{\partial}}^{n-p, n-q}\left(U_{1}\right)^{*},
$$


which we call the $\bar{\partial}$-Alexander homomorphism. Note that, although the cohomology $H_{\bar{\partial}}^{p, q}(M, M \backslash S)$ does not depend on the choice of $U_{1}$, because of the exact sequence $(3.3), H_{\bar{\partial}}^{n-p, n-q}\left(U_{1}\right)^{*}$ does depend on the choice of $U_{1}$. From the above construction, we have the following:

Proposition 3.2. If $M$ is compact, the following diagram is commutative:

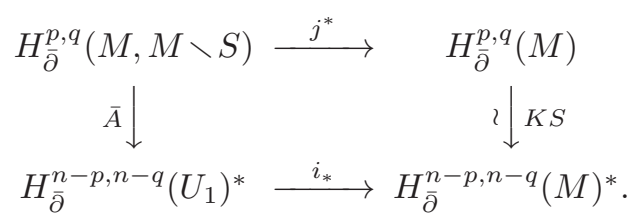

\section{Localization of Atiyah classes}

In this section we describe a general scheme for dealing with localization problems.

\subsection{Atiyah classes in the Čech-Dolbeault cohomology}

Let $M$ be a complex manifold and $\mathcal{U}=\left\{U_{0}, U_{1}\right\}$ an open covering of $M$ consisting of two open sets, so that

$$
A^{p, p}(\mathcal{U})=A^{p, p}\left(U_{0}\right) \oplus A^{p, p}\left(U_{1}\right) \oplus A^{p, p-1}\left(U_{01}\right) .
$$

For $i=0,1$, let $\nabla_{i}$ be a $(1,0)$-connection for $E$ on $U_{i}$. Then the cochain

$$
a^{p}\left(\nabla_{*}\right)=\left(a^{p}\left(\nabla_{0}\right), a^{p}\left(\nabla_{1}\right), a^{p}\left(\nabla_{0}, \nabla_{1}\right)\right)
$$

is in fact a cocycle, because of (1.4), and thus defines a class $\left[a^{p}\left(\nabla_{*}\right)\right]$ in $H_{\bar{D}}^{p, p}(\mathcal{U})$.

As in the case of Chern classes, it is not difficult to show that the class $\left[a^{p}\left(\nabla_{*}\right)\right]$ does not depend on the choice of the connections $\nabla_{i}$ and corresponds to the Atiyah class $a^{p}(E)$ via the isomorphism of Theorem 3.1 (cf. Ch. II, 8. D, of [23]).

Similarly, if $\varphi$ is a symmetric homogeneous polynomial of degree $d$, the cocycle

$$
\varphi^{A}\left(\nabla_{*}\right)=\left(\varphi^{A}\left(\nabla_{0}\right), \varphi^{A}\left(\nabla_{1}\right), \varphi^{A}\left(\nabla_{0}, \nabla_{1}\right)\right)
$$

defines a class in $H_{\bar{D}}^{d, d}(\mathcal{U})$, which corresponds to the class $\varphi^{A}(E)$ via the isomorphism of Theorem 3.1.

\subsection{Localization principle}

Let $M$ be a complex manifold of dimension $n$ and $E$ a holomorphic vector bundle of rank $\ell$ over $M$. Also, let $S$ be a closed set in $M$ and $U_{1}$ a neighborhood of $S$. Setting $U_{0}=M \backslash S$, we consider the covering $\mathcal{U}=\left\{U_{0}, U_{1}\right\}$ of $M$. Recall that for a homogeneous symmetric polynomial $\varphi$ of degree $d$, the characteristic class $\varphi^{A}(E)$ in $H_{\bar{D}}^{d, d}(\mathcal{U}) \simeq H_{\bar{\partial}}^{d, d}(M)$ is represented by the cocycle $\varphi^{A}\left(\nabla_{*}\right)$ in $A^{d, d}(\mathcal{U})$ given by (4.1). 
It often happens (see, e.g., Remark 2.12, Theorems 5.1 and 6.10 below, or $[2],[3])$ that the existence of a geometric object $\gamma$ on $U_{0}$ implies the vanishing of $\varphi\left(\left.E\right|_{U_{0}}\right)$ or of $\varphi^{A}\left(\left.E\right|_{U_{0}}\right)$, or even of the forms representing them, for some symmetric homogeneous polynomial $\varphi$. In this section we shall show that in this case we can localize the class $\varphi^{A}(E)$ at $S$.

To formalize this idea, assume that given a symmetric homogeneous polynomial $\varphi$ we can associate to $\gamma$ a class $\mathcal{C}$ of $(1,0)$-connections for $\left.E\right|_{U_{0}}$ such that

$$
\varphi^{A}(\nabla) \equiv 0
$$

for all $\nabla \in \mathcal{C}$. We shall also assume (see, e.g., Theorem 6.10) that

$$
\varphi^{A}\left(\nabla_{0}, \nabla_{1}\right) \equiv 0
$$

for all pairs $\nabla_{0}, \nabla_{1} \in \mathcal{C}$. In this case we shall say that $\varphi$ is adapted to $\gamma$, and we shall call any connection in $\mathcal{C}$ special.

Assume that $\nabla_{0}$ is special and $\varphi$ is adapted to $\gamma$. The cocycle $\varphi^{A}\left(\nabla_{*}\right)$ is then in $A^{d, d}\left(\mathcal{U}, U_{0}\right)$ and thus it defines a class in $H_{\bar{\partial}}^{d, d}(M, M \backslash S)$, which is denoted by $\varphi_{S}^{A}(E, \gamma)$. It is sent to the class $\varphi^{A}(E)$ by the canonical homomorphism $j^{*}: H_{\bar{\partial}}^{d, d}(M, M \backslash S) \rightarrow H_{\bar{\partial}}^{d, d}(M)$. It is not difficult to see that the class $\varphi_{S}^{A}(E, \gamma)$ does not depend on the choice of the special connection $\nabla_{0}$ or of the connection $\nabla_{1}$ (cf. Chapter III, Lemma 3.1 in [23]). We call $\varphi_{S}^{A}(E, \gamma)$ the localization of $\varphi^{A}(E)$ at $S$ by $\gamma$.

Suppose now $S$ is compact. Then we have the $\bar{\partial}$-Alexander homomorphism (3.4)

$$
\bar{A}: H_{\bar{\partial}}^{d, d}(M, M \backslash S) \longrightarrow H_{\bar{\partial}}^{n-d, n-d}\left(U_{1}\right)^{*} .
$$

Thus the class $\varphi_{S}^{A}(E, \gamma)$ defines a class in $H_{\bar{\partial}}^{n-d, n-d}\left(U_{1}\right)^{*}$, which we call the residue of $\gamma$ for the class $\varphi^{A}(E)$ on $U_{1}$, and denote by $\operatorname{Res}_{\varphi^{A}}\left(\gamma, E ; U_{1}\right)$.

Suppose moreover that $S$ has a finite number of connected components $\left\{S_{\lambda}\right\}_{\lambda}$. For each $\lambda$, we choose a neighborhood $U_{\lambda}$ of $S_{\lambda}$ so that $U_{\lambda} \cap U_{\mu}=\emptyset$ if $\lambda \neq \mu$. Then we have the residue $\operatorname{Res}_{\varphi^{A}}\left(\gamma, E ; U_{\lambda}\right)$ in $H_{\bar{\partial}}^{n-d, n-d}\left(U_{\lambda}\right)^{*}$ for each $\lambda$. Let $R_{\lambda}$ be a $2 n$-dimensional manifold with $C^{\infty}$ boundary in $U_{\lambda}$ containing $S_{\lambda}$ in its interior and set $R_{0 \lambda}=-\partial R_{\lambda}$. Then the residue $\operatorname{Res}_{\varphi^{A}}\left(\gamma, E ; U_{\lambda}\right)$ is represented by a functional

$$
\eta \mapsto \int_{R_{\lambda}} \varphi^{A}\left(\nabla_{1}\right) \wedge \eta+\int_{R_{0 \lambda}} \varphi^{A}\left(\nabla_{0}, \nabla_{1}\right) \wedge \eta
$$

for every $\bar{\partial}$-closed $(n-d, n-d)$-form $\eta$ on $U_{\lambda}$.

From the above considerations and Proposition 3.2, we have the following residue theorem:

Theorem 4.1. Let $E$ be a holomorphic vector bundle on a complex manifold $M$ of dimension $n$. Let $S$ be a compact subset of $M$ with a finite number of connected components $\left\{S_{\lambda}\right\}_{\lambda}$. Assume we have a geometric object $\gamma$ on $U_{0}=M \backslash S$ and a symmetric homogeneous polynomial $\varphi$ of degree $d$, adapted to $\gamma$. For each $\lambda$ choose a neighbourhood $U_{\lambda}$ of $S_{\lambda}$ so that $U_{\lambda} \cap U_{\mu}=\emptyset$ when $\lambda \neq \mu$. Then: 
1) For each connected component $S_{\lambda}$ the residue $\operatorname{Res}_{\varphi^{A}}\left(\gamma, E ; U_{\lambda}\right)$ in the dual space $H_{\bar{\partial}}^{n-d, n-d}\left(U_{\lambda}\right)^{*}$ is represented by the functional (4.2);

2) if moreover $M$ is compact, then

$$
\sum_{\lambda}\left(i_{\lambda}\right)_{*} \operatorname{Res}_{\varphi^{A}}\left(\gamma, E ; U_{\lambda}\right)=K S\left(\varphi^{A}(E)\right) \quad \text { in } H_{\bar{\partial}}^{n-d, n-d}(M)^{*},
$$

where $i_{\lambda}: U_{\lambda} \hookrightarrow M$ denotes the inclusion.

Remark 4.2. If $d=n$ and if $M$ is compact and connected, $H_{\bar{\partial}}^{n-d, n-d}(M)^{*}=$ $H_{\bar{\partial}}^{0,0}(M)^{*}$ may be identified with $\mathbb{C}$, and in this case, $\left(i_{\lambda}\right)_{*} \operatorname{Res}_{\varphi^{A}}\left(\gamma, E ; U_{\lambda}\right)$ is a complex number given by

$$
\int_{R_{\lambda}} \varphi^{A}\left(\nabla_{1}\right)+\int_{R_{0 \lambda}} \varphi^{A}\left(\nabla_{0}, \nabla_{1}\right),
$$

and $K S\left(\varphi^{A}(E)\right)$ may be expressed as $\int_{M} \varphi^{A}(E)$.

Furthermore, in this case $H_{\bar{\partial}}^{0,0}(M)^{*}=H_{0}(M, \mathbb{C})$, and $\varphi^{A}$ may be replaced by $\varphi$ (cf. Remark 1.9) so that the Atiyah residue equals the Chern residue.

We finish this section by studying what happens in the case of compact Kähler manifolds. Let $M$ be a compact Kähler manifold of dimension $n$, and $E$ a holomorphic vector bundle on $M$. We have the following commuting diagram:

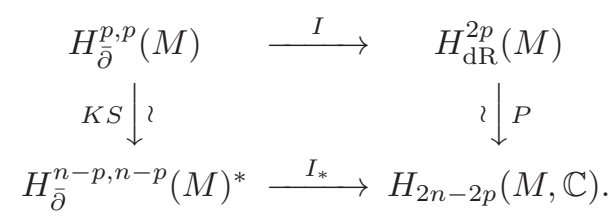

where $I$ denotes the injection given by the Hodge decomposition, $I_{*}$ the injection given by the dual decomposition, and $P$ the Poincaré isomorphism, which is given by the cap product with the fundamental cycle $[M]$.

Since $I\left(\varphi^{A}(E)\right)=\varphi(E)$ in this case (Proposition 1.10), applying $I_{*}$ to both sides of the formula in Theorem 4.12), we actually have a localization result for Chern classes:

Theorem 4.3. Let $E$ be a holomorphic vector bundle on a compact Kähler manifold $M$ of dimension $n$. Let $S$ be a compact subset of $M$ with a finite number of connected components $\left\{S_{\lambda}\right\}_{\lambda}$. Assume we have a geometric object $\gamma$ on $U_{0}=M \backslash S$ and a symmetric homogeneous polynomial $\varphi$ of degree $d$, adapted to $\gamma$. For each $\lambda$ choose a neighbourhood $U_{\lambda}$ of $S_{\lambda}$ so that $U_{\lambda} \cap U_{\mu}=\emptyset$ when $\lambda \neq \mu$. Then

$$
\sum_{\lambda} I_{*}\left(\left(i_{\lambda}\right)_{*} \operatorname{Res}_{\varphi^{A}}\left(\gamma, E ; U_{\lambda}\right)\right)=\varphi(E) \frown[M] \quad \text { in } H_{2 n-2 d}(M, \mathbb{C}) .
$$

Notice that $I_{*}\left(\left(i_{\lambda}\right)_{*} \operatorname{Res}_{\varphi^{A}}\left(\gamma, E ; U_{\lambda}\right)\right)$ is represented by a cycle $C$ such that for each closed $(2 n-2 d)$-form $\omega$, the integral $\int_{C} \omega$ is given by the right-hand side of (4.2) with $\eta$ a $\bar{\partial}$-closed $(n-d, n-d)$-form representing the $(n-d, n-d)$ component of the class $[\omega] \in H_{\mathrm{dR}}^{2 n-2 d}(M)$. 


\section{Localization by frames}

In this section we give a first example of localization of Atiyah classes following the scheme indicated in the previous section.

The starting point is the following vanishing theorem, which is a consequence of the corresponding vanishing theorem for Chern forms (see, e.g., Chapter II, Proposition 9.1 in [23]).

Theorem 5.1. Let $E$ be a holomorphic vector bundle of rank $\ell$ on a complex manifold $M$. Let $s^{(r)}=\left(s_{1}, \ldots, s_{r}\right)$ be an $r$-frame of $E$ on an open set $U \subset M$, and let $\nabla$ be an $s^{(r)}$-trivial $(1,0)$-connection for $E$ on $U$. Then

$$
a^{p}(\nabla)=0, \quad \text { on } U \text { for } p \geq \ell-r+1 .
$$

Let $S$ be a closed set in $M$ and assume we have an $r$-frame $s^{(r)}$ of $E$ on $M \backslash S$. We let $U_{0}=M \backslash S$, choose a neighborhood $U_{1}$ of $S$, and consider the covering $\mathcal{U}=\left\{U_{0}, U_{1}\right\}$ of $M$. Let $\nabla_{0}$ be an $s^{(r)}$-trivial $(1,0)$-connection for $E$ on $U_{0}$, and $\nabla_{1}$ an arbitrary $(1,0)$-connection for $E$ on $U_{1}$. The $p$-th Atiyah class $a^{p}(E)$ is represented by the Čech-Dolbeault cocycle

$$
a^{p}\left(\nabla_{*}\right)=\left(a^{p}\left(\nabla_{0}\right), a^{p}\left(\nabla_{1}\right), a^{p}\left(\nabla_{0}, \nabla_{1}\right)\right) .
$$

By Theorem 5.1, if $p \geq \ell-r+1$, we have $a^{p}\left(\nabla_{0}\right)=0$; thus $a^{p}\left(\nabla_{*}\right) \in A^{p, p}\left(\mathcal{U}, U_{0}\right)$ determines a class in $H_{\bar{\partial}}^{p, p}(M, M \backslash S)$, which we denote by $a^{p}\left(E, s^{(r)}\right)$ and call the localization of $a^{p}(E)$ by $s^{(r)}$.

Remark 5.2. If we have several $s^{(r)}$-trivial $(1,0)$-connections, we also have the vanishing of their difference form, and so $s^{(r)}$-trivial $(1,0)$-connections are special in the sense discussed in the previous section. As a consequence, the localization $a^{p}\left(E, s^{(r)}\right)$ does not depend on the choice of the $s^{(r)}$-trivial $(1,0)$-connection $\nabla_{0}$ (or of the $(1,0)$-connection $\nabla_{1}$ ); see [23].

Example 5.3. Let $C$ be a compact Riemann surface and $L$ a holomorphic line bundle over $C$. Suppose we have a meromorphic section $s$ of $L$ and let $S$ be the set of zeros and poles of $s$. The previous construction gives us the localization $a^{1}(L, s)$ in $H_{\bar{\partial}}^{1,1}(C, C \backslash S)$ of $a^{1}(L)$ in $H_{\bar{\partial}}^{1,1}(C)$. Note that $S$ consists of a finite number of points. Let $p$ be a point in $S$ and choose an open neighborhood $U$ of $p$ not containing any other point in $S$ and trivializing $L$. Let $e$ be a holomorphic frame of $L$ on $U$, and write $s=f e$ with $f$ a meromorphic function on $U$. Let $\nabla_{0}$ be the $s$-trivial connection for $L$ on $C \backslash S$ and $\nabla_{1}$ the $e$-trivial connection for $L$ on $U$. If we denote by $i$ the embedding $U \hookrightarrow C$, we have (by Theorem 4.1 and Remark 4.2)

$$
i_{*} \operatorname{Res}_{a^{1}}(L, s ; U)=\int_{R} a^{1}\left(\nabla_{1}\right)-\int_{\partial R} a^{1}\left(\nabla_{0}, \nabla_{1}\right) .
$$

But we also have $a^{1}\left(\nabla_{1}\right)=0$, and a computation gives

$$
a^{1}\left(\nabla_{0}, \nabla_{1}\right)=\frac{\sqrt{-1}}{2 \pi} \frac{d f}{f} .
$$


So

$$
i_{*} \operatorname{Res}_{a^{1}}(L, s ; U)=\frac{1}{2 \pi \sqrt{-1}} \operatorname{Res}_{p}\left(\frac{d f}{f}\right),
$$

and Theorem 4.3 yields

$$
\sum_{p \in S} \frac{1}{2 \pi \sqrt{-1}} \operatorname{Res}_{p}\left(\frac{d f}{f}\right)=\int_{C} a^{1}(L) .
$$

In particular we have recovered the classical residue formula for the Chern class, as $\int_{C} c^{1}(L)=\int_{C} a^{1}(L)$ in this case.

See [25] for another fundamental example of localized classes of this type, the "D̄-Thom class" of a holomorphic vector bundle.

\section{A Bott type vanishing theorem}

Let $M$ be a complex manifold and $E$ a complex vector bundle over $M$. If $H$ is a subbundle of the complexified tangent bundle $T_{\mathbb{R}}^{c} M$, then its dual $H^{*}$ is canonically viewed as a quotient of $\left(T_{\mathbb{R}}^{c} M\right)^{*}$. We denote by $\rho$ the canonical projection $\left(T_{\mathbb{R}}^{c} M\right)^{*} \rightarrow H^{*}$. Following [6], we give the following definition:

Definition 6.1. A partial connection for $E$ is a pair $(H, \delta)$ given by a subbundle $H$ of $T_{\mathbb{R}}^{c} M$ and a $\mathbb{C}$-linear map

$$
\delta: A^{0}(M, E) \longrightarrow A^{0}\left(M, H^{*} \otimes E\right)
$$

satisfying

$$
\delta(f s)=\rho(d f) \otimes s+f \delta(s) \quad \text { for } f \in A^{0}(M) \text { and } s \in A^{0}(M, E) .
$$

As in the case of connections, it is easy to show that a partial connection is a local operator and thus it admits locally a representation by a matrix whose entries are $C^{\infty}$ sections of $H^{*}$.

Definition 6.2. Let $(H, \delta)$ be a partial connection for $E$. We say that a connection $\nabla$ for $E$ extends $(H, \delta)$ if the diagram

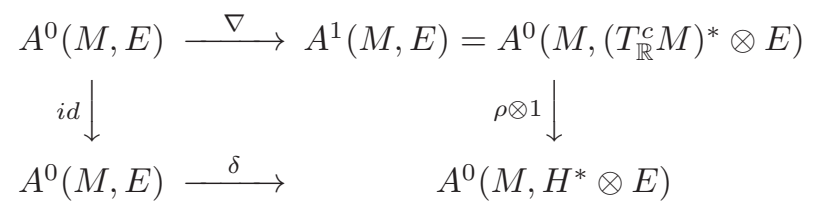

is commutative.

It is easy to see that the following lemma holds (see Lemma (2.5) in [6]).

Lemma 6.3. Any partial connection for a complex vector bundle admits an extension. 
Example 6.4. If $E$ is holomorphic, then we have the differential operator

$$
\bar{\partial}: A^{0}(M, E) \longrightarrow A^{0}\left(M, \bar{T}^{*} M \otimes E\right) .
$$

The pair $(\bar{T} M, \bar{\partial})$ is a partial connection for $E$.

The following is not difficult to prove:

Lemma $6.5([6])$. A connection $\nabla$ for a holomorphic vector bundle $E$ is of type $(1,0)$ if and only if it extends $(\bar{T} M, \bar{\partial})$.

Definition 6.6. Let $E$ be a holomorphic vector bundle over $M$. A holomorphic partial connection for $E$ is a pair $(F, \boldsymbol{\delta})$ given by holomorphic subbundle $F$ of $T M$ and a $\mathbb{C}$-linear homomorphism

$$
\delta: \mathcal{E} \longrightarrow \mathcal{F}^{*} \otimes \mathcal{E}
$$

satisfying

$$
\boldsymbol{\delta}(f s)=\rho(d f) \otimes s+f \boldsymbol{\delta}(s) \quad \text { for } f \in \mathcal{O} \text { and } s \in \mathcal{E} .
$$

We shall also say that $\boldsymbol{\delta}$ is a holomorphic partial connection along $F$.

Remark 6.7. A holomorphic partial connection $(F, \boldsymbol{\delta})$ for a holomorphic vector bundle $E$ induces a partial connection in the sense of Definition 6.1 (cf. Remark 2.2). Conversely, if $(F, \delta)$ is a $\left(C^{\infty}\right)$ partial connection such that $\delta(s)(u)$ is holomorphic wherever $s$ and $u$ are holomorphic, then it defines a holomorphic partial connection, and we shall say that $(F, \delta)$ is holomorphic.

Note that, if there is an "action" of $F$ on $E$, it naturally defines a partial connection for $E$ along $F$ (see Ch. II, 9, of [23]).

Remark 6.8. A holomorphic connection $\nabla$ on $E$ clearly gives a holomorphic partial connection $(T M, \boldsymbol{\nabla})$. The connection $\nabla$ in Remark 2.2 (that is, $\boldsymbol{\nabla}$ viewed as a $C^{\infty}$ connection) is a connection extending $(T M \oplus \bar{T} M, \boldsymbol{\nabla} \oplus \bar{\partial})$.

Definition 6.9. Let $(F, \delta)$ be a partial holomorphic connection for $E$. An $F$ connection for $E$ is a connection for $E$ extending $(F \oplus \bar{T} M, \delta \oplus \bar{\partial})$.

Using holomorphic partial connections we have a vanishing theorem generalizing Proposition 2.11:

Theorem 6.10. Let $M$ be a complex manifold of dimension $n$ and $F$ a holomorphic subbundle of rank $r$ of $T M, r \leq n$. Let $E$ be a holomorphic vector bundle over $M$ and $(F, \delta)$ a holomorphic partial connection for $E$. If $\nabla_{0}, \ldots, \nabla_{q}$ are $F$-connections for $E$, then

$$
\varphi^{A}\left(\nabla_{0}, \ldots, \nabla_{q}\right) \equiv 0
$$

for all homogeneous symmetric polynomials $\varphi$ of degree $d>n-r$.

Proof. For simplicity, we prove the theorem for the case $q=0$. The case for general $q$ follows from the construction of the difference form (see Subsection 1.2). 
Thus let $\nabla$ be an $F$-connection for $E$. Note that the problem is local; so choose a holomorphic frame $s^{(\ell)}=\left(s_{1}, \ldots, s_{\ell}\right)$ of $E$ on some open set $U$, and let $\theta$ be the connection matrix of $\nabla$ with respect to $s^{(\ell)}$. Taking a smaller $U$, if necessary, we may write $T M=F \oplus G$ for some holomorphic vector bundle $G$ of rank $n-r$ on $U$. We have the corresponding decomposition $T^{*} M=F^{*} \oplus G^{*}$. Taking, again if necessary, a smaller $U$, we can choose a holomorphic frame $u^{(r)}=\left(u_{1}, \ldots, u_{r}\right)$ of $F$ on $U$. Let $\left(u_{1}^{*}, \ldots, u_{r}^{*}\right)$ be the holomorphic frame of $F^{*}$ dual to $u^{(r)}$ and let $\left(v_{1}^{*}, \ldots, v_{n-r}^{*}\right)$ be a holomorphic frame of $G^{*}$ on $U$. Since $\nabla$ is of type $(1,0)$, each entry of $\theta$ may be written as $\sum_{j=1}^{r} a^{j} u_{j}^{*}+\sum_{k=1}^{n-r} b^{k} v_{k}^{*}$ with $a^{j}, b^{k} \in A^{0}(U)$. By definition, we have $\nabla\left(s_{i}\right)\left(u_{j}\right)=\delta\left(s_{i}\right)\left(u_{j}\right)$, which is holomorphic. Thus each $a^{j}$ is holomorphic and hence the corresponding entry of $\kappa^{1,1}=\bar{\partial} \theta$ is of the form

$$
\sum_{k=1}^{n-r} \bar{\partial} b^{k} \wedge v_{k}^{*}
$$

which yields the theorem.

Another proof of the same theorem can be given along the lines of the original Bott vanishing theorem and of Theorem 6.1 in [3]:

Second proof of Theorem 6.10. Let $\nabla$ and $T M=F \oplus G$ be chosen as in the previous proof. The curvature $K$ of $\nabla$ satisfies

$$
K(X, \bar{Z})=0
$$

for all sections $X$ of $F$ and $\bar{Z}$ of $\bar{T} M$. Hence, if

$$
\left\{u_{1}^{*}, \ldots, u_{r}^{*}, v_{1}^{*}, \ldots, v_{n-r}^{*}, d \bar{z}_{1}, \ldots, d \bar{z}_{n}\right\}
$$

is a basis of $\left(T_{\mathbb{R}}^{c} M\right)^{*}$ with respect to the decomposition $T_{\mathbb{R}}^{c} M=F \oplus G \oplus \bar{T} M$, it follows that the $(1,1)$-part of each entry of the curvature matrix of $K$ in such a frame is of the form

$$
\sum_{j=1}^{n-r} \sum_{k=1}^{n} a_{k}^{j} v_{j}^{*} \wedge d \bar{z}_{k},
$$

and again the assertion follows.

Remark 6.11. The previous vanishing theorem is an analogue of the Bott vanishing theorem for Chern forms. As shown in Theorem 6.1 of [3], under the same hypotheses we have $\varphi(\nabla)=0$ for a symmetric homogeneous polynomial $\varphi$ of degree $d>n-r+\left[\frac{r}{2}\right]$, where $[q]$ denotes the integer part of $q$.

See Section 9, Remark 9.2 below for an example where the Atiyah form vanishes but the corresponding Chern form does not.

Remark 6.12. A version of this Bott type vanishing theorem for Atiyah classes is proved in Proposition (3.3) of [5] and Proposition 5.1 of [13] by cohomological arguments (actually, in the latter the authors assume $F$ to be involutive, but involutivity is not really needed in their argument). The above theorem gives a more precise form of the vanishing theorem in the sense that it gives the vanishing at the level of forms. 
From Theorem 6.10, Remark 6.11 and Propositions 1.10 and 2.11, we have:

Theorem 6.13. Let $E$ be a holomorphic vector bundle on a complex manifold $M$. Assume that $E$ admits a holomorphic connection $\nabla$, and let $\nabla$ be corresponding $(1,0)$-connection ( $c f$. Remark 6.8). Let $\varphi$ be a a symmetric homogeneous polynomial of degree $d>0$. Then $\varphi^{A}(\nabla)=0$. Moreover if $d>\left[\frac{n}{2}\right]$, then $\varphi(\nabla)=0$. Furthermore, if $M$ is compact Kähler then $\varphi(E)=0$.

\section{Partial connection for the normal bundle of an invariant submanifold}

Let $M$ be a complex manifold. A (nonsingular holomorphic) distribution on $M$ is a holomorphic subbundle $F$ of $T M$. The rank of the distribution is the rank of $F$. In this section, we construct a partial connection for the normal bundle of an invariant submanifold of a distribution.

Let $V$ be a complex submanifold of $M$. We denote by $\mathcal{I}_{V} \subset \mathcal{O}$ the ideal sheaf of holomorphic function germs vanishing on $V$ so that $\mathcal{O}_{V}=\mathcal{O} / \mathcal{I}_{V}$ is the sheaf of germs of holomorphic functions on $V$. Denoting by $N_{V}$ the normal bundle of $V$ in $M$, we have the exact sequence

$$
\left.0 \longrightarrow T V \longrightarrow T M\right|_{V} \stackrel{\pi}{\longrightarrow} N_{V} \longrightarrow 0 .
$$

We say that a distribution $F$ on $M$ leaves $V$ invariant (or $F$ is tangent to $V$ ), if $\left.F\right|_{V} \subset T V$.

Theorem 7.1. Let $V$ be a complex submanifold of $M$. If a distribution $F$ on $M$ leaves $V$ invariant, there exists a holomorphic partial connection $\boldsymbol{\delta}$ for the normal bundle $N_{V}$ along $\left.F\right|_{V}$.

Proof. Let $x$ be a point in $V$ and take $u \in \mathcal{O}_{V}\left(\left.F\right|_{V}\right)_{x}$ and $s \in \mathcal{O}_{V}\left(N_{V}\right)_{x}$. Let $\tilde{u} \in \mathcal{F}_{x}$ and $\widetilde{s} \in \Theta_{x}$ be such that $\left.\tilde{u}\right|_{V}=u$ and $\pi\left(\left.\widetilde{s}\right|_{V}\right)=s$, where $\pi: \mathcal{O}_{V}\left(\left.T M\right|_{V}\right) \rightarrow$ $\mathcal{O}_{V}\left(N_{V}\right)$ is the natural projection. Define $\boldsymbol{\delta}: \mathcal{O}_{V}\left(N_{V}\right) \rightarrow \mathcal{O}_{V}\left(\left.F\right|_{V}\right)^{*} \otimes \mathcal{O}_{V}\left(N_{V}\right)$ by

$$
\boldsymbol{\delta}(s)(u):=\pi\left(\left.[\tilde{u}, \widetilde{s}]\right|_{V}\right) .
$$

It is easy to show that $\boldsymbol{\delta}$ does not depend on the choice of $\widetilde{s}$. As for $\tilde{u}$, let $F$ be locally generated by holomorphic sections $\tilde{v}_{1}, \ldots, \tilde{v}_{r}$ of $T M$, where $r=\operatorname{rank} F$. Choose local coordinates $\left\{z_{1}, \ldots, z_{n}\right\}$ on $M$ such that $V=\left\{z_{m+1}=\ldots=z_{n}=0\right\}$. We shall denote by $T_{k}$ any local vector field of the form $\sum_{j=1}^{m} a^{j} \frac{\partial}{\partial z_{j}}$ with $a^{j} \in \mathcal{I}_{V}^{k}$ (where clearly $\mathcal{I}_{V}^{0}=\mathcal{O}$ ); by $N_{k}$ any local vector field of the form $\sum_{j=m+1}^{n} a^{j} \frac{\partial}{\partial z_{j}}$ with $a^{j} \in \mathcal{I}_{V}^{k}$; and by $R_{k}$ any local vector field of the form $\sum_{j=1}^{n} a^{j} \frac{\partial}{\partial z_{j}}$ with $a^{j} \in \mathcal{I}_{V}^{k}$.

Since $\left.F\right|_{V} \subset T V$, it follows that $\tilde{v}_{j}=T_{0}+N_{1}+R_{2}$ for $j=1, \ldots, r$. Therefore, since the rank of $F$ and the rank of $\left.F\right|_{V}$ are the same, if

$$
u=\left.\sum_{j=1}^{r} g^{j} \tilde{v}_{j}\right|_{V}
$$


with $g^{j} \in \mathcal{O}_{V}$, then

$$
\tilde{u}=\sum_{j=1}^{r} \tilde{g}^{j} \tilde{v}_{j}
$$

with $\tilde{g}^{j} \in \mathcal{O}$ such that $\left.\tilde{g}^{j}\right|_{V}=g^{j}$. Denoting by $g^{j}$ the natural extension $\left(z_{1}, \ldots, z_{n}\right) \mapsto$ $g^{j}\left(z_{m+1}, \ldots, z_{n}\right)$, it follows that

$$
\tilde{g}^{j}-g^{j}=h^{j} \in \mathcal{I}_{V}
$$

Hence

$$
\tilde{u}=\sum_{j=1}^{r} g^{j} \tilde{v}_{j}+\sum_{j=1}^{r} h^{j} \tilde{v}_{j} .
$$

However,

$$
h^{j} \tilde{v}_{j}=h^{j}\left(T_{0}+N_{1}+R_{2}\right)=T_{1}+R_{2},
$$

and it is easy to see that this latter term does not contribute to the expression $\pi\left(\left.[\tilde{u}, \widetilde{s}]\right|_{V}\right)$. From this it follows that $\delta$ is well defined, and it is easy to check that it is a holomorphic partial connection.

Note that the above partial connection is already known for foliations (see, e.g., [18]). From Theorems 7.1 and 6.10, we have:

Corollary 7.2. Let $V$ be a complex submanifold of $M$ of dimension $m$, and let $F$ be a distribution on $M$ of rank $r \leq m$ leaving $V$ invariant. Also let $\nabla$ be a $(1,0)$-connection for $N_{V}$ extending the partial connection $\boldsymbol{\delta}$ of Theorem 7.1 . Then $\varphi^{A}(\nabla)=0$ for all symmetric homogeneous polynomials $\varphi$ of degree $d>m-r$.

We also get the following obstruction to the existence of distributions (not necessarily integrable) tangent to a given submanifold:

Corollary 7.3. Let $V$ and $F$ be as in Corollary 7.2. Then $\varphi^{A}\left(N_{V}\right)=0$ for all symmetric homogeneous polynomials $\varphi$ of degree $d>m-r$.

Moreover, if $V$ is compact Kähler then we have $\varphi\left(N_{V}\right)=0$ for all symmetric homogeneous polynomial $\varphi$ of degree $d>m-r$.

\section{Residues of singular distributions}

A general theory of singular holomorphic distributions can be developed modifying the theory for singular holomorphic foliations (see [6], and Chapter VI of [23]), by omitting the integrability condition.

Let $M$ be a complex manifold of dimension $n$. For simplicity, we assume that $M$ is connected.

Definition 8.1. A (singular) holomorphic distribution of rank $r$ on $M$ is a coherent sub- $\mathcal{O}_{M}$-module $\mathcal{F}$ of rank $r$ of $\Theta$. 
In the above, the rank of $\mathcal{F}$ is the rank of its locally free part. Note that, since $\Theta$ is locally free, the coherence of $\mathcal{F}$ here simply means that it is locally finitely generated. We call $\mathcal{F}$ the tangent sheaf of the distribution and the quotient $\mathcal{N}_{\mathcal{F}}=\Theta / \mathcal{F}$ the normal sheaf of the distribution.

The singular set $S(\mathcal{F})$ of a distribution $\mathcal{F}$ is defined to be the singular set of the coherent sheaf $\mathcal{N}_{\mathcal{F}}$ :

$$
S(\mathcal{F})=\operatorname{Sing}\left(\mathcal{N}_{\mathcal{F}}\right)=\left\{x \in M \mid \mathcal{N}_{\mathcal{F} x} \text { is not } \mathcal{O}_{x} \text {-free }\right\}
$$

Note that $\operatorname{Sing}(\mathcal{F}) \subset S(\mathcal{F})$. Away from $S(\mathcal{F})$, the sheaf $\mathcal{F}$ defines a nonsingular distribution of rank $r$.

In particular, if $\mathcal{F}$ is locally free of rank $r$, in a neighborhood of each point in $M$ it is generated on $U$ by $r$ holomorphic vector fields $v_{1}, \ldots, v_{r}$ without relations. The set $S(\mathcal{F}) \cap U$ is the set of points where the vector fields fail to be linearly independent.

Singular distributions can be dually defined in terms of the cotangent sheaf. Thus a singular distribution of corank $q$ is a rank $q$ coherent subsheaf $\mathcal{G}$ of $\Omega^{1}$. Its annihilator

$$
\mathcal{F}=\mathcal{G}^{a}=\{v \in \Theta \mid\langle v, \omega\rangle=0 \text { for all } \omega \in \mathcal{G}\}
$$

is a singular distribution of rank $r=n-q$.

Corollary 7.3 in the previous section has a slightly stronger version when the rank of the distribution is equal to the dimension of the submanifold. Namely,

Proposition 8.2. Let $V \subset M$ be a complex submanifold of dimension $m$. Let $\mathcal{F}$ be $a$ (possibly singular) holomorphic distribution of rank $m$. Assume that $\mathcal{F} \otimes \mathcal{O}_{V} \subset$ $\mathcal{O}_{V}(T V)$ and that $\Sigma=S(\mathcal{F}) \cap V$ is an analytic subset of $V$ of codimension at least 2. Then $a^{p}\left(N_{V}\right)=0$ for all $p>0$.

Moreover, if $V$ is compact Kähler then $c^{p}\left(N_{V}\right)=0$ for all $p>0$.

Proof. We shall show that there exists a holomorphic connection for $N_{V}$, then the result follows from Theorem 6.13.

By Theorem 7.1 there exists a holomorphic connection $\nabla$ for $N_{V}$ on $V \backslash \Sigma$. We are going to prove that such a connection extends holomorphically through $\Sigma$. Indeed, let $p \in \Sigma$. Let $U$ be an open neighborhood of $p$ in $V$ such that $\left.N_{V}\right|_{U}$ is trivial. Let $e_{1}, \ldots, e_{k}$ be a holomorphic frame for $\left.N_{V}\right|_{U}$ (here $k=\operatorname{dim} M-m$ ). Let $\theta$ be the connection matrix of $\nabla$ on $U \backslash \Sigma$. With respect to local coordinates $\left(z_{1}, \ldots, z_{m}\right)$ on $U$, the entries of $\theta$ are $(1,0)$-forms of the type $\sum_{j} a_{j}(z) d z_{j}$, with $a_{j}: U \backslash \Sigma \rightarrow \mathbb{C}$ holomorphic. Since $\Sigma$ has codimension at least two in $U$, Riemann's extension theorem implies that each $a_{j}$ admits a (unique) holomorphic extension to $U$. In this way we have extended $\nabla$ over $U$, and hence $N_{V}$ admits a holomorphic connection.

Now suppose $\mathcal{F}$ is a singular distribution of rank $r$ and set $U_{0}=M \backslash S$ and $S=S(\mathcal{F})$. Let $U_{1}$ be a neighborhood of $S$ in $M$ and consider the covering $\mathcal{U}=$ $\left\{U_{0}, U_{1}\right\}$. On $U_{0}$, we have a subbundle $F_{0}$ of $T M$ such that $\left.\mathcal{F}\right|_{U_{0}}=\mathcal{O}\left(F_{0}\right)$. 
Suppose $E$ is a holomorphic vector bundle on $M$ admitting a partial holomorphic connection $\left(F_{0}, \delta\right)$ on $U_{0}$. Then, choosing an $F_{0}$-connection $\nabla_{0}$ on $U_{0}$ and a $(1,0)$-connection $\nabla_{1}$ on $U_{1}$, for a symmetric homogeneous polynomial $\varphi$ of degree $d>n-r$, we have the localization $\varphi^{A}(E, \mathcal{F})$ in $H_{\bar{D}}^{d, d}\left(\mathcal{U}, U_{0}\right)$ of $\varphi^{A}(E)$ in $H_{\bar{D}}^{d, d}(\mathcal{U}) \simeq H_{\bar{\partial}}^{d, d}(M)$ and, via the $\bar{\partial}$-Alexander homomorphism, the corresponding residues.

We restate the residue theorem (Theorem 4.1) in this context:

Theorem 8.3. In the above situation, suppose $S$ has a finite number of connected components $\left\{S_{\lambda}\right\}_{\lambda}$. Then:

1) For each $\lambda$ we have the residue $\operatorname{Res}_{\varphi^{A}}\left(\mathcal{F}, E ; U_{\lambda}\right)$ in $H_{\bar{\partial}}^{n-d, n-d}\left(U_{\lambda}\right)^{*}$;

2) if $M$ is compact, then

$$
\sum_{\lambda}\left(i_{\lambda}\right)_{*} \operatorname{Res}_{\varphi^{A}}\left(\mathcal{F}, E ; U_{\lambda}\right)=K S\left(\varphi^{A}(E)\right) \quad \text { in } H_{\bar{\partial}}^{n-d, n-d}(M)^{*} .
$$

\section{An example}

In this section, we give an example of the Atiyah residue of a singular distribution on the normal bundle of an invariant submanifold.

We start with the 1-form

$$
\omega=z d x+z d y-y d z
$$

on $\mathbb{C}^{3}$ with coordinates $(x, y, z)$. It defines a corank one singular distribution on $\mathbb{C}^{3}$ with singular set $\{y=z=0\}$. As generators of its annihilator, we may take the vector fields

$$
v_{1}=y \frac{\partial}{\partial y}+z \frac{\partial}{\partial z} \quad \text { and } \quad v_{2}=\frac{\partial}{\partial x}-\frac{\partial}{\partial y} .
$$

The distribution defined by $\omega$ leaves the plane $\{z=0\}$ invariant. Note that from $\omega \wedge d \omega=-z d x \wedge d y \wedge d z$, we see that $\omega$ defines a contact structure on $\mathbb{C}^{3}$ with singular set $\{z=0\}$ (Martinet hypersurface). We will see that the first Atiyah class of the normal bundle of the (projectivized) Martinet hypersurface is localized at the singular set of the corresponding distribution.

Now we projectivize everything. Thus let $\mathbb{P}^{3}$ be the complex projective space of dimension three with homogeneous coordinates $\zeta=\left(\zeta_{0}: \zeta_{1}: \zeta_{2}: \zeta_{3}\right)$. The projective space $\mathbb{P}^{3}$ is covered by four open sets $W^{(i)}, 0 \leq i \leq 3$, given by $\zeta_{i} \neq 0$. We take the original affine space $\mathbb{C}^{3}$ as $W^{(0)}$ with $x=\zeta_{1} / \zeta_{0}, y=\zeta_{2} / \zeta_{0}$ and $z=\zeta_{3} / \zeta_{0}$.

We consider the corank one distribution $\mathcal{G}$ on $\mathbb{P}^{3}$ naturally obtained as an extension of the above:

0) On $W^{(0)}, \mathcal{G}$ is defined by $\omega_{0}=z d x+z d y-y d z$ as given before.

1) On $W^{(1)}$, we set $x_{1}=\zeta_{0} / \zeta_{1}, y_{1}=\zeta_{3} / \zeta_{1}$ and $z_{1}=\zeta_{2} / \zeta_{1}$. Then $\mathcal{G}$ is defined by

$$
\omega_{1}=-y_{1} d x_{1}-x_{1} z_{1} d y_{1}+x_{1} y_{1} d z_{1} \text {. }
$$


2) On $W^{(2)}$, we set $x_{2}=\zeta_{3} / \zeta_{2}, y_{2}=\zeta_{0} / \zeta_{2}$ and $z_{2}=\zeta_{1} / \zeta_{2}$. Then $\mathcal{G}$ is defined by

$$
\omega_{2}=-y_{2} d x_{2}-x_{2} z_{2} d y_{2}+x_{2} y_{2} d z_{2}
$$

3) On $W^{(3)}$, we set $x_{3}=\zeta_{2} / \zeta_{3}, y_{3}=\zeta_{1} / \zeta_{3}$ and $z_{3}=\zeta_{0} / \zeta_{3}$. Then $\mathcal{G}$ is defined by

$$
\omega_{3}=z_{3} d x_{3}+z_{3} d y_{3}-y_{3} d z_{3}
$$

Note that $\omega_{i}=\left(\zeta_{j} / \zeta_{i}\right)^{3} \omega_{j}$ in $W^{(i)} \cap W^{(j)}$ so that the conormal sheaf of the distribution $\mathcal{G}$ is locally free of rank one and, as a line bundle, it is -3 times the hyperplane bundle on $\mathbb{P}^{3}$. Let $\mathcal{F}=\mathcal{G}^{a}$ be the annihilator of $\mathcal{G}$, which defines a singular distribution of rank two on $\mathbb{P}^{3}$. The singular set $S(\mathcal{F})$ of $\mathcal{F}$, which coincides with that of $\mathcal{G}$, has three irreducible components $S_{1}=\left\{\zeta_{2}=\zeta_{3}=0\right\}$, $S_{2}=\left\{\zeta_{0}=\zeta_{3}=0\right\}$ and $S_{3}=\left\{\zeta_{0}=\zeta_{1}=0\right\}$. We have a subbundle $F_{0}$ of rank 2 of $T \mathbb{P}^{3}$ on $\mathbb{P}^{3} \backslash S(\mathcal{F})$ defining $\mathcal{F}$ away from $S(\mathcal{F})$.

The distribution $\mathcal{F}$ leaves the hyperplane $V=\left\{\zeta_{3}=0\right\} \simeq \mathbb{P}^{2}$ invariant and we work on $V$. In fact the distribution $\mathcal{F}$ also leaves invariant the singular hypersurface $\left\{\zeta_{0} \zeta_{3}=0\right\}$, which contains the whole $S(\mathcal{F})$. This case is treated in [26].

Thus we consider the singular distribution $\mathcal{F}_{V}=\mathcal{F} \otimes \mathcal{O}_{V}$ on $V$, whose singular set $S$ is given by $S=S(\mathcal{F}) \cap V=S_{1} \cup S_{2}$. We let $P=(0: 1: 0: 0)$, which is the intersection point of $S_{1}$ and $S_{2}$. The restriction of the bundle $F_{V, 0}=\left.F_{0}\right|_{V}$ defines $\mathcal{F}_{V}$ on $U_{0}=V \backslash S$. As is shown in Section 7 , the normal bundle $N_{V}$ of $V$ in $\mathbb{P}^{3}$ admits a partial connection along $F_{V, 0}$ on $U_{0}$ and the first Atiyah class $a^{1}\left(N_{V}\right)$ is localized near $S$ and yields an "Atiyah residue".

Note that, although a priori the first Chern class $c^{1}\left(N_{V}\right)$ is not localized in this context, it has the "Atiyah localization" and the "Atiyah residue", since it coincides with $a^{1}\left(N_{V}\right), V$ being compact Kähler (see Remarks 9.2 and 9.5 below).

To describe the localization more precisely, we need the Čech-Dolbeault cohomology theory for coverings involving more than two open sets, as $S$ is singular in our case. We briefly recall what is needed in our case.

Let $U_{0}=V \backslash S$ be as above and let $U_{1}, U_{2}$ and $U_{3}$ be neighborhoods of $S_{1} \backslash\{P\}$, $S_{2} \backslash\{P\}$ and $P$ in $V$, respectively, such that $U_{1} \subset W^{(0)}, U_{2} \subset W^{(2)}$ and $U_{3} \subset W^{(1)}$. Then $\mathcal{U}=\left\{U_{0}, \ldots, U_{3}\right\}$ is a covering of $V$ and $\mathcal{U}^{\prime}=\left\{U_{1}, U_{2}, U_{3}\right\}$ is a covering of $U^{\prime}=U_{1} \cup U_{2} \cup U_{3}$, which is an open neighborhood of $S$ in $V$. Letting $U_{i j}=U_{i} \cap U_{j}$ and $U_{i j k}=U_{i} \cap U_{j} \cap U_{k}$, we set

$$
A^{p, q}(\mathcal{U}):=\oplus_{i} A^{p, q}\left(U_{i}\right) \oplus_{i, j} A^{p, q-1}\left(U_{i j}\right) \oplus_{i, j, k} A^{p, q-2}\left(U_{i j k}\right),
$$

where in the first sum, $0 \leq i \leq 3$, in the second, $0 \leq i<j \leq 3$ and in the third, $0 \leq i<j<k \leq 3$. The differential operator

$$
\bar{D}: A^{p, q}(\mathcal{U}) \longrightarrow A^{p, q+1}(\mathcal{U})
$$

is defined by

$$
\bar{D}\left(\sigma_{i}, \sigma_{i j}, \sigma_{i j k}\right)=\left(\bar{\partial} \sigma_{i}, \sigma_{j}-\sigma_{i}-\bar{\partial} \sigma_{i j}, \sigma_{j k}-\sigma_{i k}+\sigma_{i j}+\bar{\partial} \sigma_{i j k}\right) .
$$


The $q$-th cohomology of the complex $\left(A^{p, *}(\mathcal{U}), \bar{D}\right)$ is the Čech-Dolbeault cohomology $H_{\bar{D}}^{p, q}(\mathcal{U})$ of $\mathcal{U}$ of type $(p, q)$, which is shown to be canonically isomorphic to the Dolbeault cohomology $H_{\bar{\partial}}^{p, q}(V)$ of $V$ (see Theorem 3.1).

Likewise we have the cohomology $H_{\bar{D}}^{p, q}\left(\mathcal{U}^{\prime}\right)$ of the complex $\left(A^{p, *}\left(\mathcal{U}^{\prime}\right), \bar{D}\right)$ by omitting $U_{0}$ in the above.

Also, setting $A^{p, q}\left(\mathcal{U}, U_{0}\right)=\left\{\sigma \in A^{p, q}(\mathcal{U}) \mid \sigma_{0}=0\right\}$, we have the relative cohomology $H_{\bar{D}}^{p, q}\left(\mathcal{U}, U_{0}\right)$, which we also denote by $H_{\bar{\partial}}^{p, q}(V, V \backslash S)$.

The Atiyah classes are defined in the Čech-Dolbeault cohomology as in Subsection 4.1, taking a $(1,0)$-connection on each open set and making use of difference forms. In our case, the first Atiyah class $a^{1}\left(N_{V}\right)$ is represented by the cocycle $a^{1}\left(\nabla_{*}\right)$ in

$$
A^{1,1}(\mathcal{U})=\oplus_{i} A^{1,1}\left(U_{i}\right) \oplus_{i<j} A^{1,0}\left(U_{i j}\right)
$$

(note that $A^{p, q-2}\left(U_{i j k}\right)=0$ in $(9.2)$, if $\left.(p, q)=(1,1)\right)$ given by

$$
a^{1}\left(\nabla_{*}\right)=\left(a^{1}\left(\nabla_{i}\right), a^{1}\left(\nabla_{i}, \nabla_{j}\right)\right)
$$

with $\nabla_{i}$ a $(1,0)$-connection on $U_{i}$. If we take an $F_{V, 0}$-connection as $\nabla_{0}$, we have $a^{1}\left(\nabla_{0}\right)=0$ (see Theorem 6.10). Hence $a^{1}\left(\nabla_{*}\right)$ is in $A^{1,1}\left(\mathcal{U}, U_{0}\right)$ and defines the localization $a^{1}\left(N_{V}, \mathcal{F}_{V}\right)$ in $H_{\bar{D}}^{1,1}\left(\mathcal{U}, U_{0}\right)$.

Recall that $V$ is defined by $\zeta_{3}=0$ in $\mathbb{P}^{3}$. Thus, in $W^{(0)}$ it is defined by $z=0$ with $(x, y)$ coordinates on $W^{(0)} \cap V\left(\supset U_{1}\right)$; in $W^{(2)}$ it is defined by $x_{2}=0$ with $\left(y_{2}, z_{2}\right)$ coordinates on $W^{(2)} \cap V\left(\supset U_{2}\right)$; and in $W^{(1)}$ it is defined by $y_{1}=0$ with $\left(x_{1}, z_{1}\right)$ coordinates on $W^{(1)} \cap V\left(\supset U_{3}\right)$.

Proposition 9.1. Let $\mathcal{F}$ be the singular distribution on $\mathbb{P}^{3}$ as above. It leaves the hyperplane $V$ given by $\zeta_{3}=0$ invariant. We have the localization $a^{1}\left(N_{V}, \mathcal{F}_{V}\right)$ in $H_{\bar{D}}^{1,1}\left(\mathcal{U}, U_{0}\right)$ of $a^{1}\left(N_{V}\right)$ in $H_{\bar{D}}^{1,1}(\mathcal{U})=H_{\bar{\partial}}^{1,1}(V)$. By a suitable choice of connections $\nabla_{i}$, it is represented by the Čech-Dolbeault cocycle $a^{1}\left(\nabla_{*}\right)=\left(a^{1}\left(\nabla_{i}\right), a^{1}\left(\nabla_{i}, \nabla_{j}\right)\right)$ given by

$$
\begin{array}{ll}
a^{1}\left(\nabla_{i}\right)=0,0 \leq i \leq 3, & a^{1}\left(\nabla_{0}, \nabla_{1}\right)=\frac{\sqrt{-1}}{2 \pi} \frac{d x+d y}{y} \\
a^{1}\left(\nabla_{0}, \nabla_{2}\right)=\frac{\sqrt{-1}}{2 \pi}\left(z_{2} \frac{d y_{2}}{y_{2}}-d z_{2}\right), & a^{1}\left(\nabla_{0}, \nabla_{3}\right)=-\frac{\sqrt{-1}}{2 \pi}\left(\frac{d x_{1}}{x_{1} z_{1}}-\frac{d z_{1}}{z_{1}}\right) \\
a^{1}\left(\nabla_{1}, \nabla_{2}\right)=\frac{\sqrt{-1}}{2 \pi} \frac{d y_{2}}{y_{2}}, & a^{1}\left(\nabla_{1}, \nabla_{3}\right)=\frac{\sqrt{-1}}{2 \pi} \frac{d x_{1}}{x_{1}}, \\
a^{1}\left(\nabla_{2}, \nabla_{3}\right)=\frac{\sqrt{-1}}{2 \pi} \frac{d z_{1}}{z_{1}} . &
\end{array}
$$

Proof. By taking an $F_{V, 0}$-connection for $N_{V}$ on $U_{0}$ as $\nabla_{0}$, we have $a^{1}\left(\nabla_{i}\right)=0$ as above. We have the exact sequence

$$
\left.0 \longrightarrow T V \longrightarrow T \mathbb{P}^{3}\right|_{V} \stackrel{\pi}{\longrightarrow} N_{V} \longrightarrow 0 \text {. }
$$


On each of $U_{1}, U_{2}$ and $U_{3}$, the bundle $N_{V}$ is trivial and we may take $\nu_{1}=\pi\left(\frac{\partial}{\partial z}\right)$, $\nu_{2}=\pi\left(\frac{\partial}{\partial x_{2}}\right)$ and $\nu_{3}=\pi\left(\frac{\partial}{\partial y_{1}}\right)$, respectively, as a frame of $N_{V}$. Let $\nabla_{i}$ be the connection trivial with respect to $\nu_{i}$. Then we have $a^{1}\left(\nabla_{i}\right)=0,1 \leq i \leq 3$.

To compute the difference forms $a^{1}\left(\nabla_{i}, \nabla_{j}\right)$, we first make the following observation (cf. Subsection 1.2). Let $\theta_{i}$ be the connection matrix (form, in this case) of $\nabla_{i}$ with respect to some holomorphic frame $\nu$ of $N_{V}$. Then, since the $\theta_{i}$ 's are of type $(1,0)$,

$$
a^{1}\left(\nabla_{i}, \nabla_{j}\right)=c^{1}\left(\nabla_{i}, \nabla_{j}\right)=\frac{\sqrt{-1}}{2 \pi}\left(\theta_{j}-\theta_{i}\right) .
$$

Moreover, if $\tilde{\nu}=a \nu$ is another holomorphic frame and if the $\tilde{\theta}_{i}$ 's are corresponding connection forms, we have (cf. (1.1))

$$
\tilde{\theta}_{i}=\theta_{i}+\frac{d a}{a}
$$

We first compute $a^{1}\left(\nabla_{0}, \nabla_{1}\right)$. For this, we find the connection forms $\theta_{0}$ and $\theta_{1}$ of $\nabla_{0}$ and $\nabla_{1}$ with respect to the frame $\nu_{1}$. Since $\theta_{1}=0$, we only need to find $\theta_{0}$. Note that $U_{01} \subset W^{(0)}$, where we may take the vector fields $v_{1}$ and $v_{2}$ in (9.1) as generators of $\mathcal{F}$. We set

$$
u_{1}=\left.v_{1}\right|_{V}=y \frac{\partial}{\partial y} \quad \text { and } \quad u_{2}=\left.v_{2}\right|_{V}=\frac{\partial}{\partial x}-\frac{\partial}{\partial y}
$$

Since $\theta_{0}$ is of type $(1,0)$, we may write as $\theta_{0}=f d x+g d y$. Then, on the one hand we have $\nabla_{0}\left(\nu_{1}\right)\left(u_{1}\right)=y g \cdot \nu_{1}$ and $\nabla_{0}\left(\nu_{1}\right)\left(u_{2}\right)=(f-g) \cdot \nu_{1}$. On the other hand by definition,

$$
\nabla_{0}\left(\nu_{1}\right)\left(u_{1}\right)=\pi\left(\left.\left[y \frac{\partial}{\partial y}+z \frac{\partial}{\partial z}, \frac{\partial}{\partial z}\right]\right|_{V}\right)=-\nu_{1}
$$

and

$$
\nabla_{0}\left(\nu_{1}\right)\left(u_{2}\right)=\pi\left(\left.\left[\frac{\partial}{\partial x}-\frac{\partial}{\partial y}, \frac{\partial}{\partial z}\right]\right|_{V}\right)=0
$$

Hence we get

$$
\theta_{0}=-\frac{d x+d y}{y}
$$

which gives the expression for $a^{1}\left(\nabla_{0}, \nabla_{1}\right)$ by $(9.4)$.

Similar computations show that the connection forms of $\nabla_{0}$ with respect to the frames $\nu_{2}$ and $\nu_{3}$ are, respectively, $-z_{2} \frac{d y_{2}}{y_{2}}+d z_{2}$ and $\frac{d x_{1}}{x_{1} z_{1}}-\frac{d z_{1}}{z_{1}}$, which give the expressions for $a^{1}\left(\nabla_{0}, \nabla_{2}\right)$ and $a^{1}\left(\nabla_{0}, \nabla_{3}\right)$.

Finally the relations $\nu_{2}=\frac{1}{y_{2}} \nu_{1}, \nu_{3}=\frac{1}{x_{1}} \nu_{1}$ and $\nu_{3}=\frac{1}{z_{1}} \nu_{2}$ give the expressions for $a^{1}\left(\nabla_{1}, \nabla_{2}\right), a^{1}\left(\nabla_{1}, \nabla_{3}\right)$ and $a^{1}\left(\nabla_{2}, \nabla_{3}\right)$ by $(9.5)$. 
Remark 9.2. From the above, we see that the curvature form of $\nabla_{0}$ with respect to $\nu_{1}$ is given by

$$
\kappa_{0}=d \theta_{0}+\theta_{0} \wedge \theta_{0}=-\frac{d x \wedge d y}{y^{2}} .
$$

Since it has no $(1,1)$-component, there holds $a^{1}\left(\nabla_{0}\right)=0$, while $c^{1}\left(\nabla_{0}\right)=\frac{\sqrt{-1}}{2 \pi} \kappa_{0}$ does not vanish.

We now try to find the corresponding residue. For this, we first consider the cup product in our case. Recalling (9.2) and (9.3), it is the pairing

$$
A^{1,1}(\mathcal{U}) \times A^{1,1}(\mathcal{U}) \longrightarrow A^{2,2}(\mathcal{U})
$$

given by

$$
\left(\sigma_{i}, \sigma_{i j}, 0\right) \smile\left(\tau_{i}, \tau_{i j}, 0\right)=\left(\sigma_{i} \wedge \tau_{i}, \sigma_{i} \wedge \tau_{i j}+\sigma_{i j} \wedge \tau_{j},-\sigma_{i j} \wedge \tau_{j k}\right) .
$$

This induces a pairing $H_{\bar{D}}^{1,1}(\mathcal{U}) \times H_{\bar{D}}^{1,1}(\mathcal{U}) \longrightarrow H_{\bar{D}}^{2,2}(\mathcal{U})$, which followed by integration $\int_{V}: H_{\bar{D}}^{2,2}(\mathcal{U}) \simeq H_{\bar{\partial}}^{2,2}(V) \longrightarrow \mathbb{C}$ defines the Kodaira-Serre duality.

In the relative case, we have $\sigma_{0}=0$ and the above cup product involves only $\left(\tau_{i}, \tau_{i j}\right)$ with $i \geq 1$. Hence we have the pairing

$$
A^{1,1}\left(\mathcal{U}, U_{0}\right) \times A^{1,1}\left(\mathcal{U}^{\prime}\right) \longrightarrow A^{2,2}\left(\mathcal{U}, U_{0}\right)
$$

This in turn induces the pairing

$$
H_{\bar{D}}^{1,1}\left(\mathcal{U}, U_{0}\right) \times H_{\bar{D}}^{1,1}\left(\mathcal{U}^{\prime}\right) \longrightarrow H_{\bar{D}}^{2,2}\left(\mathcal{U}, U_{0}\right)
$$

which, followed by integration, defines the $\bar{\partial}$-Alexander homomorphism

$$
\bar{A}: H_{\bar{D}}^{1,1}\left(\mathcal{U}, U_{0}\right) \longrightarrow H_{\bar{D}}^{1,1}\left(\mathcal{U}^{\prime}\right)^{*}
$$

and we have a commutative diagram as in Proposition 3.2, to which we return below (see (9.9)).

We examine the $\bar{\partial}$-Alexander homomorphism more closely. We take a "system of honeycomb cells" $\left(R_{i}\right)$ adapted to $\mathcal{U}$, which will be given explicitly below. For a class $[\sigma]$ in $H_{\bar{D}}^{1,1}\left(\mathcal{U}, U_{0}\right), \sigma=\left(\sigma_{i}, \sigma_{i j}\right)$, the image of $[\sigma]$ under $\bar{A}$ is a functional assigning to each class $[\tau]$ in $H_{\bar{D}}^{1,1}\left(\mathcal{U}^{\prime}\right), \tau=\left(\tau_{i}, \tau_{i j}\right)$, the integral

$$
\begin{aligned}
\int_{V} \sigma \smile \tau= & \sum_{1 \leq i \leq 3}\left(\int_{R_{i}} \sigma_{i} \wedge \tau_{i}+\int_{R_{0 i}} \sigma_{0 i} \wedge \tau_{i}\right) \\
& +\sum_{1 \leq i<j \leq 3}\left(\int_{R_{i j}} \sigma_{i} \wedge \tau_{i j}+\sigma_{i j} \wedge \tau_{j}-\int_{R_{0 i j}} \sigma_{0 i} \wedge \tau_{i j}\right) .
\end{aligned}
$$

In the above, each $R_{i}$ has the same orientation as $V$. We set $R_{i j}=R_{i} \cap R_{j}=$ $\partial R_{i} \cap \partial R_{j}$, which has the same orientation as $\partial R_{i}$ (opposite the orientation of $\partial R_{j}$ ) and $R_{0 i j}=R_{0} \cap R_{i j}=\partial R_{0} \cap \partial R_{i j}$, which has the same orientation as $\partial R_{0 i}$. 
In fact, the right-hand side of (9.6) can be reduced by choosing Stein open sets as the $U_{i}, i=1,2,3$, which is possible (for example, we may take as $U_{1}$ a tubular neighborhood of $S_{1} \backslash\{P\}$ in $V \cap W^{(0)}$ containing $R_{1}$, or even the whole $\left.V \cap W^{(0)} \simeq \mathbb{C}^{2}\right)$.

Lemma 9.3. If we choose $U_{i}, 1 \leq i \leq 3$, to be Stein, we may represent every class in $H_{\bar{D}}^{1,1}\left(\mathcal{U}^{\prime}\right)$ by a cocycle of the form $\bar{\xi}=\left(0, \xi_{i j}\right)$.

Proof. From $\bar{D} \tau=0$, we have $\bar{\partial} \tau_{i}=0,1 \leq i \leq 3$. Since each $U_{i}$ is Stein, there exist a $(1,0)$-form $\rho_{i}$ such that $\tau_{i}=\bar{\partial} \rho_{i}$. If we set $\xi=\left(0, \xi_{i j}\right)$ with

$$
\xi_{i j}=\tau_{i j}+\rho_{i}-\rho_{j}
$$

Then we have $\tau=\xi+\bar{D} \rho, \rho=\left(\rho_{i}, 0\right)$.

If we use the representative as above, the right-hand side of (9.6) becomes

$$
\sum_{1 \leq i<j \leq 3}\left(\int_{R_{i j}} \sigma_{i} \wedge \xi_{i j}-\int_{R_{0 i j}} \sigma_{0 i} \wedge \xi_{i j}\right) .
$$

Recall that the residue $\operatorname{Res}_{a^{1}}\left(\mathcal{F}_{V}, N_{V} ; U^{\prime}\right)$ of $\mathcal{F}_{V}$ with respect to $a^{1}$ for $N_{V}$ on $U^{\prime}$ is the image of the localization $a^{1}\left(N_{V}, \mathcal{F}_{V}\right)$.

Proposition 9.4. If we choose connections $\nabla_{i}$ as in Proposition 9.1, and a representative $\xi$ of each class in $H^{1,1}\left(\mathcal{U}^{\prime}\right)$ as in Lemma 9.3, then the residue $\operatorname{Res}_{a^{1}}\left(\mathcal{F}_{V}, N_{V} ; U^{\prime}\right)$ is the functional assigning to $[\xi]$ the value

$$
-\sum_{1 \leq i<j \leq 3} \int_{R_{0 i j}} a^{1}\left(\nabla_{0}, \nabla_{i}\right) \wedge \xi_{i j} .
$$

Proof. The proposition follows from $a^{1}\left(\nabla_{i}\right)=0$ and (9.7).

The domains of integration $R_{0 i j}$ can be given explicitly, for example, as follows. Let $\delta$ be positive number with $\delta^{2}<1$, and set

$$
\begin{aligned}
& R_{3}=\left\{\left.\zeta \in V|| \zeta_{0}\right|^{2}+\left|\zeta_{2}\right|^{2} \leq \delta^{2}\left|\zeta_{1}\right|^{2}\right\}, \\
& R_{2}=\left\{\left.\zeta \in V|| \zeta_{0}\right|^{2} \leq \delta^{2}\left|\zeta_{2}\right|^{2}\right\} \backslash \operatorname{Int} R_{3}, \\
& R_{1}=\left\{\left.\zeta \in V|| \zeta_{2}\right|^{2} \leq \delta^{2}\left|\zeta_{0}\right|^{2}\right\} \backslash \operatorname{Int} R_{3}, \\
& R_{0}=U_{0} \backslash\left(\bigcup_{i=1}^{3} \operatorname{Int} R_{i} \bigcup_{1 \leq i<j \leq 3} \operatorname{Int} R_{i j}\right) .
\end{aligned}
$$

From $\delta<1$, we see that $R_{12}=\emptyset$ and thus $R_{012}=\emptyset$. We first express $R_{013}$ explicitly. As a set, it is given by

$$
|y|=\delta, \quad 1+|y|^{2}=\delta^{2}|x|^{2} \quad \text { and } \quad z=0 .
$$

Setting $\delta^{\prime}=\frac{\sqrt{1+\delta^{2}}}{\delta}$, we have

$$
R_{013}=\left\{(x, y)|| x\left|=\delta^{\prime},\right| y \mid=\delta\right\},
$$


oriented so that $\arg x \wedge \arg y$ is negative. Similarly we have

$$
R_{023}=\left\{\left(y_{2}, z_{2}\right)|| y_{2}|=\delta,| z_{2} \mid=\delta^{\prime}\right\},
$$

which is oriented so that $\arg y_{2} \wedge \arg z_{2}$ is positive.

Now we consider the commutative diagram

$$
\begin{gathered}
H_{\bar{D}}^{1,1}\left(\mathcal{U}, U_{0}\right) \stackrel{j^{*}}{\longrightarrow} H_{\bar{D}}^{1,1}(\mathcal{U}) \simeq H_{\bar{\partial}}^{1,1}(V)=H_{\bar{\partial}}^{1,1}\left(\mathbb{P}^{2}\right) \simeq H^{2}\left(\mathbb{P}^{2}, \mathbb{C}\right) \\
\downarrow_{\bar{A}_{V}} \\
H_{\bar{D}}^{1,1}\left(\mathcal{U}^{\prime}\right)^{*} \stackrel{i_{*}}{\longrightarrow} H_{\bar{D}}^{1,1}(\mathcal{U})^{*} \simeq H_{\bar{\partial}}^{1,1}(V)^{*}=H_{\bar{\partial}}^{1,1}\left(\mathbb{P}^{2}\right)^{*} \simeq H_{2}\left(\mathbb{P}^{2}, \mathbb{C}\right) .
\end{gathered}
$$

The normal bundle $N_{V}$ of $V$ in $\mathbb{P}^{3}$ is isomorphic to the hyperplane bundle $H_{2}$ on $V=\mathbb{P}^{2}$. Since $\mathbb{P}^{2}$ is compact Kähler, we know that the first Atiyah class $a^{1}\left(N_{V}\right)$ in $H_{\bar{\partial}}^{1,1}(V)=H^{2}\left(\mathbb{P}^{2}, \mathbb{C}\right) \simeq \mathbb{C}$ coincides with the first Chern class $c^{1}\left(N_{V}\right)=c^{1}\left(H_{2}\right)$, the generator of the cohomology.

We try to find $i_{*} \operatorname{Res}_{c^{1}}\left(\mathcal{F}, N_{V} ; S\right)$ and verify the Residue Theorem 4.1. Recall that the isomorphism $H_{\bar{\partial}}^{1,1}\left(\mathbb{P}^{2}\right) \longrightarrow H_{\bar{D}}^{1,1}(\mathcal{U})$ is induced by $\tau \mapsto\left(\tau_{i}, \tau_{i j}\right)=(\tau, 0)$ (cf. Theorem 3.1). Also note that $H_{\bar{\partial}}^{1,1}\left(\mathbb{P}^{2}\right) \simeq \mathbb{C}$, which is generated by the class of

$$
\tau_{0}=\frac{\sqrt{-1}}{2 \pi} \partial \bar{\partial} \log \|\zeta\|^{2}
$$

(see, e.g., [14]). For $\tau_{0}$ we may take, as $\rho_{i}$ in the proof of Lemma 9.3, the forms

$$
\begin{aligned}
& \rho_{1}=-\frac{\sqrt{-1}}{2 \pi} \frac{\bar{x} d x+\bar{y} d y}{1+|x|^{2}+|y|^{2}}, \quad \rho_{2}=-\frac{\sqrt{-1}}{2 \pi} \frac{\bar{y}_{2} d y_{2}+\bar{z}_{2} d z_{2}}{1+\left|y_{2}\right|^{2}+\left|z_{2}\right|^{2}}, \\
& \rho_{3}=-\frac{\sqrt{-1}}{2 \pi} \frac{\bar{x}_{1} d x_{1}+\bar{z}_{1} d z_{1}}{1+\left|x_{1}\right|^{2}+\left|z_{1}\right|^{2}},
\end{aligned}
$$

and we compute

$$
\xi_{13}=\rho_{1}-\rho_{3}=-\frac{\sqrt{-1}}{2 \pi} \frac{d x}{x}, \quad \xi_{23}=\rho_{2}-\rho_{3}=-\frac{\sqrt{-1}}{2 \pi} \frac{d z_{2}}{z_{2}} .
$$

Thus, to the canonical generator $\left[\tau_{0}\right]$, the residue assigns the value

$$
\begin{aligned}
-\int_{R_{013}} a^{1}\left(\nabla_{0}, \nabla_{1}\right) \wedge \xi_{13}-\int_{R_{023}} a^{1}\left(\nabla_{0}, \nabla_{2}\right) \wedge \xi_{23} \\
=\left(\frac{\sqrt{-1}}{2 \pi}\right)^{2}\left\{\int_{R_{013}}\left(\frac{d x+d y}{y}\right) \wedge \frac{d x}{x}+\int_{R_{023}}\left(z_{2} \frac{d y_{2}}{y_{2}}-d z_{2}\right) \wedge \frac{d z_{2}}{z_{2}}\right\} \\
=-\left(\frac{\sqrt{-1}}{2 \pi}\right)^{2} \int_{R_{013}} \frac{d x \wedge d y}{x y}=1
\end{aligned}
$$

(see (9.8)), as expected.

The above computation appears to suggest that the residue is concentrated on $S_{1}$.

Remark 9.5. Although the first Chern class $c^{1}\left(N_{V}\right)$ is not localized as a Chern class (see Remark 9.2), it has the "Atiyah localization" and the "Atiyah residue". 


\section{References}

[1] Abate, M.: The residual index and the dynamics of holomorphic maps tangent to the identity. Duke Math. J. 107 (2001), no. 1, 173-207.

[2] Abate, M., Bracci, F. and Tovena, F.: Index theorems for holomorphic selfmaps. Ann. of Math. (2) 159 (2004), no. 2, 819-864.

[3] Abate, M., Bracci, F. and Tovena, F.: Index theorems for holomorphic maps and foliations. Indiana Univ. Math. J. 57 (2008), no. 7, 2999-3048.

[4] Aтіяaн, M.: Complex analytic connections in fibre bundles. Trans. Amer. Math. Soc. 85 (1957), 181-207.

[5] Baum, P. And Bott, R.: On the zeroes of meromorphic vector-fields. In Essays on Topology and related topics (Memoires dédiés à George de Rham), 29-47. Springer, New York 1970.

[6] Baum, P. And Bott, R.: Singularities of holomorphic foliations. J. Differential Geometry 7 (1972), 279-342.

[7] Вотт, R.: Lectures on characteristic classes and foliations. In Lectures on Algebraic and Differential Topology (Second Latin American School in Math., Mexico City, 1971), 1-94. Lecture Notes in Mathematics 279, Springer, Berlin, 1972.

[8] Bracci, F. And Tovena, F.: Residual indices of holomorphic maps relative to singular curves of fixed points on surfaces. Math. Z. 242 (2002), no. 3, 481-490.

[9] Brasselet, J.P., Seade, J. And Suwa, T.: Vector fields on singular varieties. Lecture Notes in Mathematics 1987, Springer-Verlag, Berlin, 2009.

[10] Brunella, M.: Birational geometry of foliations. Monografías de Matemática, Instituto de Matemática Pura e Aplicada (IMPA), Rio de Janeiro, 2000.

[11] Calaque, D. And van den Bergh, M.: Hochschild cohomology and Atiyah classes. Adv. Math. 224 (2010), no. 5, 1839-1889.

[12] CAmacho, C. AND SAD, P.: Invariant varieties through singularities of holomorphic vector fields. Ann. of Math. (2) 115 (1982), no. 3, 579-595.

[13] Carrell, J. B. And Lieberman, D. I.: Vector fields and Chern numbers. Math. Ann. 225 (1977), no. 3, 263-273.

[14] Griffiths, P. And Harris, J.: Principles of algebraic geometry. Pure and Applied Mathematics, Wiley-Interscience, New York, 1978.

[15] Kapranov, M.: Rozansky-Witten invariants via Atiyah classes. Compositio Math. 115 (1999), no. 1, 71-113.

[16] Lehmann, D.: Systèmes d'alvéoles et intégration sur le complexe de Čech-de Rham. Publications de l'IRMA 23, Nº VI, Université de Lille I, 1991.

[17] Lehmann, D.: Résidus des sous-variétés invariantes d'un feuilletage singulier. Ann. Inst. Fourier (Grenoble) 41 (1991), no. 1, 211-258.

[18] Lehmann, D. And Suwa, T.: Residues of holomorphic vector fields relative to singular invariant subvarieties. J. Differential Geom. 42 (1995), no. 1, 165-192.

[19] Marín, D. et Mattei, J. F.: Monodromy and topological classification of germs of holomorphic foliations. Ann. Sci. Éc. Norm. Supér. (4) 45 (2012), no. 3, 405-445.

[20] Markarian, N.: The Atiyah class, Hochschild cohomology and the Riemann-Roch theorem. J. Lond. Math. Soc. (2) 79 (2009), no. 1, 129-143. 
[21] Milnor, J. and Stasheff, J.: Characteristic classes. Annals of Mathematics Studies 76, Princeton University Press. Princeton, N.Y.; University of Tokyo Press, Tokyo, 1974.

[22] Perrone, C.: Extendable cohomologies for complex analytic varieties. Math. Ann. 345 (2009), no. 1, 83-132.

[23] SuwA, T.: Indices of vector fields and residues of singular holomorphic foliations. Actualités Mathématiques, Hermann, Paris, 1998.

[24] SuwA, T.: Residue theoretical approach to intersection theory. In Real and complex singularities, 207-261. Contemp. Math. 459, Amer. Math. Soc., Providence, RI, 2008.

[25] SuwA, T.: Čech-Dolbeault cohomology and the $\bar{\partial}$-Thom class. In Singularities (Niigata-Toyama 2007), 321-340. Adv. Stud. Pure Math. 56, Math. Soc. Japan, Tokyo, 2009.

[26] Suwa, T.: Residues of singular holomorphic distributions. In Singularities in Geometry and Topology (Strasbourg 2009), 207-247. IRMA Lectures in Mathematics and Theoretical Physics 20, European Mathematical Society, 2012.

Received July 6, 2011.

Marco Abate: Dipartimento di Matematica, Università di Pisa, Largo Bruno Pontecorvo 5, 56127 Pisa, Italy.

E-mail: abate@dm.unipi.it

Filippo Bracci: Dipartimento di Matematica, Università di Roma "Tor Vergata", Via della Ricerca Scientifica 1, 00133 Roma, Italy.

E-mail: fbracci@mat.uniroma2.it

TAtsuo Suwa: Department of Mathematics, Hokkaido University, Sapporo 060-0810, Japan.

E-mail: tsuwa@sci.hokudai.ac.jp

Francesca Tovena: Dipartimento di Matematica, Università di Roma "Tor Vergata", Via della Ricerca Scientifica 1, 00133 Roma, Italy.

E-mail: tovena@mat.uniroma2.it 